\title{
One-shot quantum state redistribution and quantum Markov chains
}

\author{
Anurag Anshu* \\ Shima Bab Hadiashar ${ }^{\dagger}$ \\ UC Berkeley \\ U. Waterloo \\ Rahul Jain $\ddagger$ \\ Ashwin Nayak ${ }^{\dagger}$ \\ Dave Touchette $\S$ \\ U. Waterloo \\ U. Sherbrooke
}

\begin{abstract}
We revisit the task of quantum state redistribution in the one-shot setting, and design a protocol for this task with communication cost in terms of a measure of distance from quantum Markov chains. More precisely, the distance is defined in terms of quantum max-relative entropy and quantum hypothesis testing entropy.

Our result is the first to operationally connect quantum state redistribution and quantum Markov chains, and can be interpreted as an operational interpretation for a possible one-shot analogue of quantum conditional mutual information. The communication cost of our protocol is lower than all previously known ones and asymptotically achieves the well-known rate of quantum conditional mutual information. Thus, our work takes a step towards the important open question of near-optimal characterization of the one-shot quantum state redistribution.
\end{abstract}

\section{Introduction}

The connection between conditional mutual information and Markov chains has led to a rich body of results in classical computer science and information theory. It is well known that for any tripartite distribution $P^{R B C}$,

$$
\mathrm{I}(R: C \mid B)_{P}=\min _{Q^{R B C} \in \mathrm{MC}_{R-B-C}} \mathrm{D}\left(P^{R B C} \| Q^{R B C}\right),
$$

${ }^{*}$ Department of EECS and Challenge Institute for Quantum Computation, University of California, Berkeley, USA. Simons Institute for the Theory of Computing, Berkeley, California, USA. Email: anuraganshu@berkeley.edu . Research supported by the NSF QLCI program through grant number OMA-2016245. Part of the work was done when the author was affiliated to the Department of Combinatorics and Optimization \& the Institute for Quantum Computing, University of Waterloo and the Perimeter Institute for Theoretical Physics, Waterloo, Canada.

${ }^{\dagger}$ Department of Combinatorics and Optimization, and Institute for Quantum Computing, University of Waterloo, 200 University Ave. W., Waterloo, ON, N2L 3G1, Canada. Email: \{sbabhadi, ashwin.nayak\}@uwaterloo.ca . Research supported in part by NSERC Canada. SBH is also supported by an Ontario Graduate Scholarship.

${ }^{\ddagger}$ Department of Computer Science, and Center for Quantum Technologies, National University of Singapore, 21 Lower Kent Ridge Rd, Singapore 119077. Email: rahul@comp.nus.edu.sg.

${ }^{\S}$ Department of Computer Science, and Institut Quantique, Université de Sherbrooke, 2500 Boulevard de l'Université, Sherbrooke, QC J1K 2R1, Canada. Email: dave.touchette@usherbrooke.ca. 


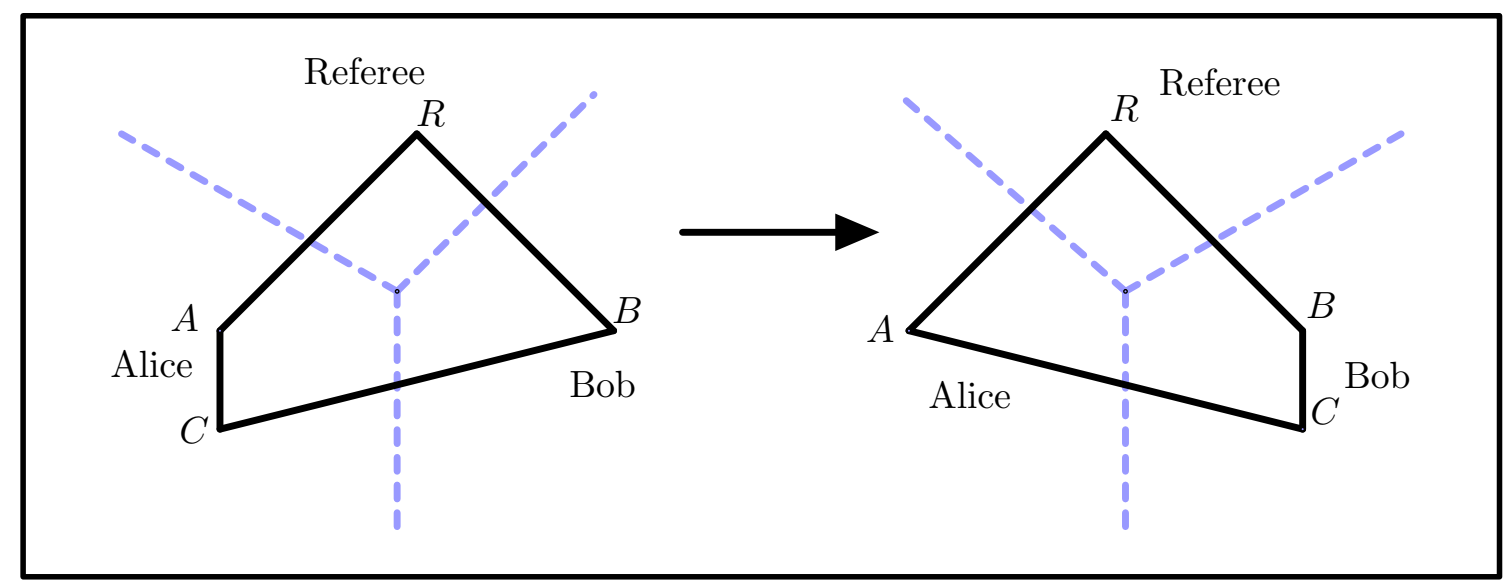

Figure 1: An illustration of quantum state redistribution.

where $\mathrm{MC}_{R-B-C}$ is the set of Markov distributions $Q$, i.e., those that satisfy $\mathrm{I}(R: C \mid B)_{Q}=0$. In fact, one can choose a distribution $Q$ achieving the minimum above with $Q^{R B}=P^{R B}, Q^{B C}=P^{B C}$. In the quantum case, the above identity fails drastically. For an example presented in Ref. [11] (see also Ref. [15, Section VI]), the right-hand side is a constant, whereas the left-hand side approaches zero as the system size increases. Given this, it is natural to ask if there is an extension of the classical identity to the quantum case. This is shown to be true in a sense that for any tripartite quantum state $\psi^{R B C}$, it holds that

$$
\mathrm{I}(R: C \mid B)_{\psi}=\min _{\sigma^{R B C} \in \mathrm{QMC}_{R-B-C}}\left(\mathrm{D}\left(\psi^{R B C} \| \sigma^{R B C}\right)-\mathrm{D}\left(\psi^{B C} \| \sigma^{B C}\right)\right)
$$

where $\mathrm{QMC}_{R-B-C}$ is the set of quantum states $\sigma$ satisfying $\mathrm{I}(R: C \mid B)_{\sigma}=0, \psi^{R B}=\sigma^{R B}[10$ ]. A proof is provided in Section 2.2, Lemma 2.8. The difference between the quantum and the classical expressions can now be understood as follows. For the classical case, the closest Markov chain $Q$ to a distribution $P$ (in relative entropy) satisfies the aforementioned relations $Q^{R B}=P^{R B}$ and $Q^{B C}=P^{B C}$. Thus, the second relative entropy term vanishes in Eq. (1.1). In quantum case, due to monogamy of entanglement we cannot in general ensure that $\sigma^{B C}=\psi^{B C}$. Thus, the quantum relative entropy distance to quantum Markov chains can be bounded away from the quantum conditional mutual information.

In this work, we prove a one-shot analogue of Eq. (1.1). This is achieved in an operational manner, by showing that a one-shot analogue of the right-hand side in Eq. (1.1) is the achievable communication cost in quantum state redistribution of $|\psi\rangle^{R A B C}$, a purification of $\psi^{R B C}$. In the task of quantum state redistribution, a pure quantum state $|\psi\rangle^{R A B C}$ known to two parties, Alice and Bob, is shared between Alice (who has registers $A C$ ), Bob (who has $B$ ), and Referee (who has $R$ ). Additionally, Alice and Bob may share an arbitrary (pure) entangled state. The goal is to transmit the content of register $C$ to Bob using a communication protocol involving only Alice and Bob, in such a way that all correlations, including those with Referee, are approximately preserved. (See Figure 1 for an illustration of state redistribution.) Given a quantum state $\phi^{R B C}$, we identify a natural subset of Markov extensions of $\phi^{R B}$, which we denote by $\mathrm{ME}_{R-B-C}^{\epsilon, \phi}$ and define formally at the end of Section 2.1. We establish the following result.

Theorem 1.1. For any pure quantum state $|\psi\rangle^{R A B C}$, the quantum communication cost of redistributing the register $C$ from Alice (who initially holds $A C$ ) to Bob (who initially holds B) with 
error $10 \sqrt{\epsilon}$ is at most

$$
\frac{1}{2} \min _{\psi^{\prime} \in \mathrm{B}^{\epsilon}\left(\psi^{R B C}\right)} \min _{\sigma^{R B C} \in \mathrm{ME}_{R-B-C}^{\epsilon^{2} / 4, \psi^{\prime}}}\left[\mathrm{D}_{\max }\left(\psi^{\prime R B C} \| \sigma^{R B C}\right)-\mathrm{D}_{\mathrm{H}}^{\epsilon}\left(\psi^{\prime B C} \| \sigma^{B C}\right)\right]+\mathrm{O}\left(\log \frac{1}{\epsilon}\right) .
$$

The difference between minimizing over the set $\mathrm{ME}_{R-B-C}^{\epsilon^{2} / 4, \psi^{\prime}}$ versus $\mathrm{QMC}_{R-B-C}$ appears to be minor, and is best understood from the definitions in Section 2.1. We believe the above result can be stated in terms of a minimization over all of $\mathrm{QMC}_{R-B-C}$. As far as we know, this result is the first that operationally connects the cost of quantum state redistribution to Markov chains (even in the asymptotic i.i.d. setting). The best previously known achievable one-shot bound for the communication cost of state redistribution, namely,

$$
\frac{1}{2} \inf _{\sigma^{C}} \inf _{\psi^{\prime} \in \mathrm{B}^{\epsilon}\left(\psi^{R B C}\right)}\left(\mathrm{D}_{\max }\left(\psi^{\prime R B C} \| \psi^{\prime R B} \otimes \sigma^{C}\right)-\mathrm{D}_{\mathrm{H}}^{\epsilon^{2}}\left(\psi^{\prime B C} \| \psi^{\prime B} \otimes \sigma^{C}\right)\right)+\log \frac{1}{\epsilon^{2}},
$$

when the state $|\psi\rangle^{R A B C}$ is redistributed with error $\mathrm{O}(\epsilon)$ was due to Anshu, Jain, and Warsi [5]. Note that $\sigma^{C}:=\psi^{\prime C}$ is a nearly optimal solution for Eq. (1.2) as discussed in Ref. [12], and the product state $\psi^{\prime R B} \otimes \psi^{\prime C}$ is a Markov state in the set $\mathrm{ME}_{R-B-C}^{\epsilon^{2} / 4, \psi^{\prime}}$. So, the bound in Theorem 1.1 is smaller than Eq. (1.2) in the sense that the minimization is over a larger set. In the special case where $\psi^{R B C}$ is a quantum Markov chain, our protocol has near-zero communication. This feature is not present in other protocols and their communication may be as large as $(1 / 2) \log |C|$.

\section{Techniques}

The protocol we design is most easily understood by considering a folklore protocol for redistributing quantum Markov states. In the case that $\psi^{R B C}$ is a Markov state, its purification $|\psi\rangle^{R A B C}$ can be transformed through local isometry operators $V_{A}: A \rightarrow A^{R} J^{\prime} A^{C}$ and $V_{B}: B \rightarrow B^{R} J B^{C}$ into the following:

$$
V_{A} \otimes V_{B}|\psi\rangle^{R A B C}=\sum_{j} \sqrt{p(j)}\left|\psi_{j}\right\rangle^{R A^{R} B^{R}} \otimes|j j\rangle^{J J^{\prime}} \otimes\left|\psi_{j}\right\rangle^{A^{C} B^{C} C}
$$

The existence of isometries $V_{A}$ and $V_{B}$ is a consequence of the special structure of quantum Markov states proved by Hayden, Josza, Petz, and Winter [14. Note that after the above transformation, conditioned on registers $J$ and $J^{\prime}$, systems $R A^{R} B^{R}$ are decoupled from systems $A^{C} C B^{C}$. So using the embezzling technique due to van Dam and Hayden [27], conditioned on $J$ and $J^{\prime}$, Alice and Bob can first embezzle-out systems $A^{C} C B^{C}$ and then embezzle-in the same systems such that at the end the global state is close to the state in Eq. (1.3) and system $C$ is with Bob. This protocol incurs no communication; see Fig. 2 for an illustration.

The protocol we design is a more sophisticated version of the above protocol. The key technique underlying our protocol is a reduction procedure using embezzling quantum states, that allows us to use a protocol due to Anshu, Jain, and Warsi [5] as a subroutine. Let $\sigma^{R B C}$ be a quantum Markov extension of $\psi^{R B}$. The reduction procedure is a method which decouples $C$ from $R B$ when applied to $\sigma^{R B C}$, while preserving $\psi^{R B}$ when applied to $\psi^{R B C}$.

To elaborate further, consider an example where $\psi^{R B C}$ is the GHZ state $\frac{1}{\sqrt{d}} \sum_{j=1}^{d}|j\rangle^{R}|j\rangle^{B}|j\rangle^{C}$. In this case, the closest Markov extension $\sigma^{R B C}$ of $\psi^{R B}$ is $\frac{1}{d} \sum_{j=1}^{d}|j\rangle\left\langle\left. j\right|^{R} \otimes \mid j\right\rangle\left\langle\left. j\right|^{B} \otimes \mid j\right\rangle\left\langle\left. j\right|^{C}\right.$. A naive 


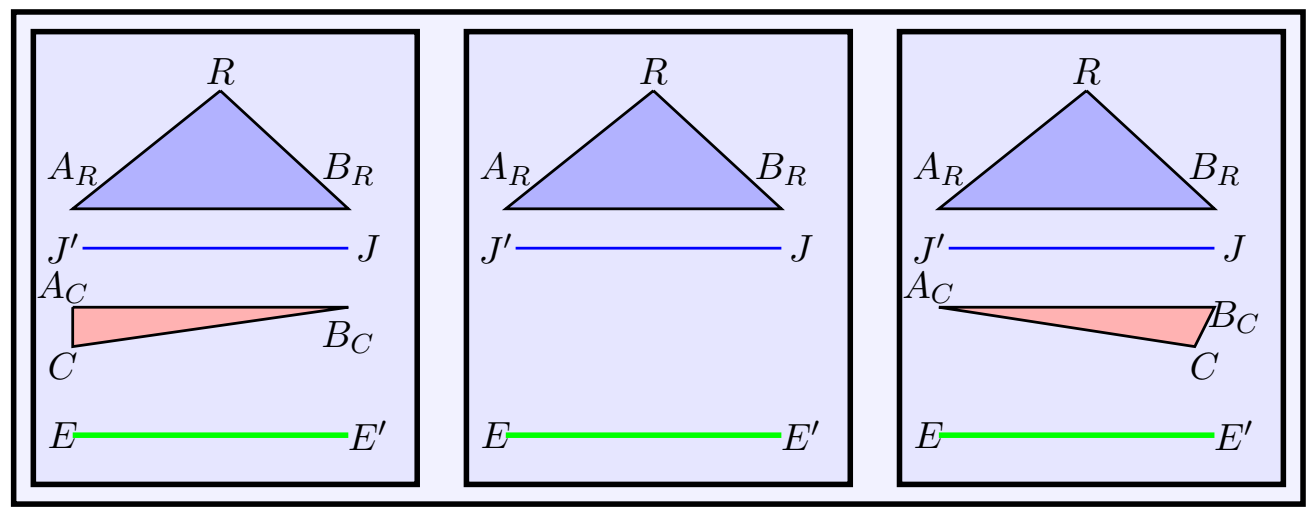

Figure 2: An illustration of the zero-cost protocol for redistributing Markov states. Left: Registers $R A^{R} B^{R} J J^{\prime} A^{C} C B^{C}$ are in the state given in Eq. (1.3) and registers $E$ and $E^{\prime}$ contain Alice and Bob's share of an embezzling state, respectively. Middle: Using embezzling registers, Alice and Bob jointly embezzled out registers $A^{C} C B^{C}$ via local unitary operations. Right: Using embezzling registers, conditioned on $J$ and $J^{\prime}$, Alice and Bob embezzled in $\left|\psi_{j}\right\rangle^{A^{C} C B^{C}}$ such that registers $C$ and $B^{C}$ are with Bob and register $A^{C}$ is with Alice. This step also only contains local unitary operations and no communication.

way to decouple register $C$ from registers $R B$ in $\sigma^{R B C}$ is to coherently erase register $C$ conditioned on register $B$. However, the same operation applied to $\psi^{R B C}$ changes $\psi^{R B}$. To overcome this problem, first, we coherently measure register $B$ by adding a maximally entangled state $|\Psi\rangle^{T T^{\prime}}$ and making another "copy" of $|j\rangle^{B}$ in $\Psi^{T}$. The copying is done by applying a distinct Heisenberg-Weyl operator to the state $\Psi^{T}$, for each $j \in[d]$. This operation measures register $B$ in $\psi^{R B C}$, keeps $\sigma^{R B C}$ unchanged, and leaves $\Psi^{T}$ in tensor product with registers $R B$ in both $\psi$ and $\sigma$. Then, conditioned on register $B$, we can coherently erase register $C$ in $\sigma^{R B C}$; this operation applied to $\psi$ does not change the state $\psi^{R B}$.

For a general state $\psi^{R B C}$ with quantum Markov extension $\sigma^{R B C}$, the isometry operator $V_{B}$ can be used to transform $\sigma^{R B C}$ to the classical-quantum state $\sum_{j} p(j) \sigma_{j}^{R B^{R}} \otimes|j\rangle\left\langle\left. j\right|^{J} \otimes \sigma_{j}^{B^{C} C}\right.$. However, we encounter an additional issue here: it may not be possible to unitarily transform all $\sigma_{j}^{B^{C} C}$ to a fixed state since the spectrum of $\sigma_{j}^{B^{C} C}$ is not necessarily the same for all $j \in[d]$. So we first flatten each $\sigma_{j}^{B^{C} C}$ through a unitary procedure. This task can be achieved via the technique of coherent flattening via embezzlement due to Anshu and Jain [3]. After flattening, the dimension of the support of systems $B^{C} C$ no more depends on $j$ and so the states in registers $B^{C} C$ can be all rotated to a flat state over a fixed subspace. Hence, $B^{C} C$ gets decoupled from $R B^{R} J$ in the state $\sigma$. Finally, to keep $\psi^{R B}$ unchanged, we regenerate the system $B^{C}$ via a standard embezzling technique similar to the protocol in Fig. 2, 


\section{Preliminaries}

\subsection{Mathematical notation and background}

For a thorough introduction to basics of quantum information, we refer the reader to the book by Watrous [28]. In this section, we briefly review the notation and some results that we use in this article.

For the sake of brevity, we denote the set $\{1,2, \ldots, k\}$ by $[k]$. We denote physical quantum systems ("registers") with capital letters, like $A, B$ and $C$. The state space corresponding to a register is a finite-dimensional Hilbert space. We denote (finite dimensional) Hilbert spaces by capital script letters like $\mathcal{H}$ and $\mathcal{K}$, and the Hilbert space corresponding to a register $A$ by $\mathcal{H}^{A}$. We sometimes refer to the space corresponding to the register $A$ by the name of the register.

We use the Dirac notation, i.e., "ket" and "bra", for unit vectors and their adjoints, respectively. We denote the set of all linear operators on Hilbert space $\mathcal{H}$ by $\mathrm{L}(\mathcal{H})$, the set of all positive semidefinite operators by $\operatorname{Pos}(\mathcal{H})$, the set of all unitary operators by $\mathrm{U}(\mathcal{H})$, and the set of all quantum states (or "density operators") over $\mathcal{H}$ by $\mathrm{D}(\mathcal{H})$. The identity operator on space $\mathcal{H}$ or register $A$, is denoted by $\mathbb{1}^{\mathcal{H}}$ or $\mathbb{1}^{A}$, respectively. Similarly, we use superscripts to indicate the registers on which an operator acts. We say a positive semi-definite operator $M \in \operatorname{Pos}(\mathcal{H})$ is a measurement operator if $M \preceq \mathbb{1}^{\mathcal{H}}$, where $\preceq$ denotes Lowener order for Hermitian operators.

We denote quantum states by lowercase Greek letters like $\rho, \sigma$. We use the notation $\rho^{A}$ to indicate that register $A$ is in quantum state $\rho$. We denote the partial trace over register $A$ by $\operatorname{Tr}_{A}$. We say $\rho^{A B}$ is an extension of $\sigma^{A}$ if $\operatorname{Tr}_{B}\left(\rho^{A B}\right)=\sigma^{A}$. A purification of a quantum state $\rho$ is an extension of $\rho$ with rank one. For the Hilbert space $\mathbb{C}^{S}$ for some set $S$, we refer to the basis $\{|x\rangle: x \in S\}$ as the canonical basis for the space. We say the register $X$ is classical in a quantum state $\rho^{X B}$ if $\rho^{X B}$ is block-diagonal in the canonical basis of $X$, i.e., $\rho^{X B}=\sum_{x} p(x)|x\rangle\left\langle\left. x\right|^{X} \otimes \rho_{x}^{B}\right.$ for some probability distribution $p$ on $X$. For a non-trivial register $B$, we say $\rho^{X B}$ is a classical-quantum state if $X$ is classical in $\rho^{X B}$. We say a unitary operator $U^{A B} \in \mathrm{U}\left(\mathcal{H}^{A} \otimes \mathcal{H}^{B}\right)$ is read-only on register $A$ if it is block-diagonal in the canonical basis of $A$, i.e., $U^{A B}=\sum_{a}|a\rangle\left\langle\left. a\right|^{A} \otimes U_{a}^{B}\right.$ where each $U_{a}^{B}$ is a unitary operator.

The trace norm (Schatten 1 norm) of an operator $M \in \mathrm{L}(\mathcal{H})$ is the sum of its singular values and we denote it by $\|M\|_{1}$. The trace distance between $\rho$ and $\sigma$ is induced by trace norm. The following theorem is a well-known property of trace norm (see, e.g., [28, Theorem 3.4, page 128]).

Theorem 2.1 (Holevo-Helstrom). For any pair of quantum states $\rho, \sigma \in \mathrm{D}(\mathcal{H})$,

$$
\|\rho-\sigma\|_{1}=2 \max \{|\operatorname{Tr}(\Pi \rho)-\operatorname{Tr}(\Pi \sigma)|: \Pi \preceq \mathbb{1}, \Pi \in \operatorname{Pos}(\mathcal{H})\} .
$$

Lemma 2.2 (Gentle Measurement [29, 19]). Let $\epsilon \in[0,1], \rho \in \mathrm{D}(\mathcal{H})$ and $\Pi \in \operatorname{Pos}(\mathcal{H})$ be a measurement operator such that $\operatorname{Tr}(\Pi \rho) \geq 1-\epsilon$. Then,

$$
\left\|\frac{\Pi \rho \Pi}{\operatorname{Tr}(\Pi \rho)}-\rho\right\|_{1} \leq 2 \sqrt{\epsilon} .
$$

The fidelity between two sub-normalized states $\rho$ and $\sigma$ is defined as

$$
\mathrm{F}(\rho, \sigma):=\operatorname{Tr} \sqrt{\sqrt{\rho} \sigma \sqrt{\rho}}+\sqrt{(1-\operatorname{Tr}(\rho))(1-\operatorname{Tr}(\sigma))} .
$$


Fidelity can be used to define a useful metric called the purified distance [13, 23] between quantum states:

$$
\mathrm{P}(\rho, \sigma) \quad:=\sqrt{1-\mathrm{F}(\rho, \sigma)^{2}} .
$$

Purified distance and trace distance are related to each other as follows (see, e.g., 28, Theorem 3.33, page 161]):

Theorem 2.3 (Fuchs and van de Graaf inequality). For any pair of quantum states $\rho, \sigma \in \mathrm{D}(\mathcal{H})$,

$$
1-\sqrt{1-\mathrm{P}(\rho, \sigma)^{2}} \leq \frac{1}{2}\|\rho-\sigma\|_{1} \leq \mathrm{P}(\rho, \sigma) .
$$

For a quantum state $\rho \in \mathrm{D}(\mathcal{H})$ and $\epsilon \in[0,1]$, we define

$$
\mathrm{B}^{\epsilon}(\rho):=\{\widetilde{\rho} \in \mathrm{D}(\mathcal{H}): \mathrm{P}(\rho, \widetilde{\rho}) \leq \epsilon\}
$$

as the ball of quantum states that are within purified distance $\epsilon$ of $\rho$. Note that in some works, the states in the set $\mathbf{B}^{\epsilon}(\rho)$ are allowed to be sub-normalized. But here, we require the states in the ball to have trace equal to one.

Theorem 2.4 (Uhlmann [26]). Consider quantum states $\rho^{A}, \sigma^{A} \in \mathrm{D}\left(\mathcal{H}^{A}\right)$. Suppose $|\xi\rangle^{A B},|\theta\rangle^{A B} \in$ $\mathrm{D}\left(\mathcal{H}^{A} \otimes \mathcal{H}^{B}\right)$ are arbitrary purifications of $\rho^{A}$ and $\sigma^{A}$, respectively. Then, there exists some unitary operator $V^{B} \in \mathrm{U}\left(\mathcal{H}^{B}\right)$ such that

$$
\mathrm{P}\left(|\xi\rangle^{A B},\left(\mathbb{1} \otimes V^{B}\right)|\theta\rangle^{A B}\right)=\mathrm{P}\left(\rho^{A}, \sigma^{A}\right) .
$$

Let $\rho \in \mathrm{D}(\mathcal{H})$ be a quantum state over the Hilbert space $\mathcal{H}$. The von Neumann entropy of $\rho$ is defined as

$$
\mathrm{S}(\rho) \quad:=-\operatorname{Tr}(\rho \log \rho)
$$

This coincides with Shannon entropy for a classical state. The relative entropy of two quantum states $\rho, \sigma \in \mathrm{D}(\mathcal{H})$ is defined as

$$
\mathrm{D}(\rho \| \sigma):=\operatorname{Tr}(\rho(\log \rho-\log \sigma)),
$$

when $\operatorname{supp}(\rho) \subseteq \operatorname{supp}(\sigma)$, and is $\infty$ otherwise. The max-relative entropy of $\rho$ with respect to $\sigma$ is defined as

$$
\mathrm{D}_{\max }(\rho \| \sigma):=\min \left\{\lambda: \rho \leq 2^{\lambda} \sigma\right\},
$$

when $\operatorname{supp}(\rho) \subseteq \operatorname{supp}(\sigma)$, and is $\infty$ otherwise. The following proposition bounds purified distance in terms of max-relative entropy. It is a special case of the monotonicity of minimal quantum $\alpha$-Rényi divergence in $\alpha$ (see, e.g., [21, Corollary 4.2, page 56]).

Proposition 2.5. Let $\mathcal{H}$ be a Hilbert space, and let $\rho, \sigma \in \mathrm{D}(\mathcal{H})$ be quantum states over $\mathcal{H}$. It holds that

$$
\mathrm{P}(\rho, \sigma) \leq \sqrt{1-2^{-\mathrm{D}_{\max }(\rho \| \sigma)}}
$$


The above property also implies the Pinsker inequality. For $\epsilon \in[0,1]$, the $\epsilon$-smooth max-relative entropy of $\rho$ with respect to $\sigma$ is defined as

$$
\mathrm{D}_{\text {max }}^{\epsilon}(\rho \| \sigma):=\min _{\rho^{\prime} \in \mathrm{B}^{\epsilon}(\rho)} \mathrm{D}_{\max }\left(\rho^{\prime} \| \sigma\right) .
$$

For $\epsilon \in[0,1]$, the $\epsilon$-hypothesis testing relative entropy of $\rho$ with respect to $\sigma$ is defined as

$$
\mathrm{D}_{\mathrm{H}}^{\epsilon}(\rho \| \sigma):=\sup _{0 \preceq \Pi \preceq \mathbb{1}, \operatorname{Tr}(\Pi \rho) \geq 1-\epsilon} \log \left(\frac{1}{\operatorname{Tr}(\Pi \sigma)}\right) .
$$

Smooth max-relative entropy and hypothesis relative entropy both converge to relative entropy in the asymptotic and i.i.d. setting [22, 20, 8. The following proposition gives upper and lower bounds for the convergence of these quantities for finite $n$ which are tight up to second order.

Theorem 2.6 ([24], [17]). Let $\epsilon \in(0,1)$ and $n$ be an integer. Consider quantum states $\rho, \sigma \in \mathrm{D}(\mathcal{H})$. Define $\mathrm{V}(\rho \| \sigma)=\operatorname{Tr}\left(\rho(\log \rho-\log \sigma)^{2}\right)-(\mathrm{D}(\rho \| \sigma))^{2}$ and $\Phi(x)=\int_{-\infty}^{x} \frac{\exp \left(-x^{2} / 2\right)}{\sqrt{2 \pi}} d x$. It holds that

$$
\mathrm{D}_{\max }^{\epsilon}\left(\rho^{\otimes n} \| \sigma^{\otimes n}\right)=n \mathrm{D}(\rho \| \sigma)-\sqrt{n \mathrm{~V}(\rho \| \sigma)} \Phi^{-1}\left(\epsilon^{2}\right)+\mathrm{O}(\log n)-\mathrm{O}(\log (1-\epsilon)),
$$

and

$$
\mathrm{D}_{\mathrm{H}}^{\epsilon}\left(\rho^{\otimes n} \| \sigma^{\otimes n}\right)=n \mathrm{D}(\rho \| \sigma)+\sqrt{n \mathrm{~V}(\rho \| \sigma)} \Phi^{-1}(\epsilon)+\mathrm{O}(\log n) .
$$

Note that Eq. (2.1) has an additional $\mathrm{O}(\log (1-\epsilon))$ term as compared to the original statement in Ref. 24] because we only allow the normalized states in $\mathrm{B}^{\epsilon}(\rho)$. We also need the following property due to Anshu, Berta, Jain, and Tomamichel [1, Theorem 2]. The original statement involves a minimization over all $\sigma_{B}$ on both sides of the inequality, but the proof works for any $\sigma_{B}$.

Theorem 2.7 ([1], Theorem 2). Let $\epsilon, \delta \in(0,1)$ such that $0 \leq 2 \epsilon+\delta \leq 1$. Consider quantum states $\sigma^{B} \in \mathrm{D}\left(\mathcal{H}^{B}\right)$ and $\rho^{A B} \in \mathrm{D}\left(\mathcal{H}^{A B}\right)$. We have

$$
\inf _{\substack{\bar{\rho} \in \mathrm{B}^{2 \epsilon+\delta}\left(\rho^{A B}\right) \\ \bar{\rho}^{A}=\rho^{A}}} \mathrm{D}_{\max }\left(\bar{\rho}^{A B} \| \rho^{A} \otimes \sigma^{B}\right) \leq \mathrm{D}_{\max }^{\epsilon}\left(\rho^{A B} \| \rho^{A} \otimes \sigma^{B}\right)+\log \frac{8+\delta^{2}}{\delta^{2}} .
$$

Suppose that $\rho^{A B} \in \mathrm{D}\left(\mathcal{H}^{A} \otimes \mathcal{H}^{B}\right)$ is the joint state of registers $A$ and $B$, then the mutual information of $A$ and $B$ is denoted by

$$
\mathrm{I}(A: B)_{\rho}:=\mathrm{D}\left(\rho^{A B} \| \rho^{A} \otimes \rho^{B}\right) .
$$

When the state is clear from the context, the subscript $\rho$ may be omitted. Let $\rho^{R B C} \in \mathrm{D}\left(\mathcal{H}^{R B C}\right)$ be a tripartite quantum state. The conditional mutual information of $R$ and $C$ given $B$ is defined as

$$
\mathrm{I}(R: C \mid B) \quad:=\mathrm{I}(R B: C)-\mathrm{I}(B: C) .
$$

For the state $\rho^{A B} \in \mathrm{D}\left(\mathcal{H}^{A} \otimes \mathcal{H}^{B}\right)$, the max-information register $B$ has about register $A$ is defined as

$$
\mathrm{I}_{\max }(A: B)_{\rho}:=\min _{\sigma^{B} \in \mathrm{D}\left(\mathcal{H}^{B}\right)} \mathrm{D}_{\max }\left(\rho^{A B} \| \rho^{A} \otimes \sigma^{B}\right) .
$$

For $\epsilon \in[0,1]$, the $\epsilon$-smooth max-information register $B$ has about register $A$ in the state $\rho^{A B} \in$ $\mathrm{D}\left(\mathcal{H}^{A} \otimes \mathcal{H}^{B}\right)$ is defined as

$$
\mathrm{I}_{\max }^{\epsilon}(A: B)_{\rho}:=\min _{\rho^{\prime} \in \mathrm{B}^{\epsilon}\left(\rho^{A B}\right)} \mathrm{I}_{\max }(A: B)_{\rho^{\prime}} .
$$




\subsection{Quantum Markov states}

A tripartite quantum state $\sigma^{R B C} \in \mathrm{D}\left(\mathcal{H}^{R B C}\right)$ is called a quantum Markov state if there exists a quantum operation $\Lambda: \mathrm{L}\left(\mathcal{H}^{B}\right) \rightarrow \mathrm{L}\left(\mathcal{H}^{B C}\right)$ such that $(\mathbb{1} \otimes \Lambda)\left(\sigma^{R B}\right)=\sigma^{R B C}$, equivalently, if $\mathrm{I}(R$ : $C \mid B)=0$. This is the quantum analogue of the notion of Markov chains for classical registers. Classical registers $Y X M$ form a Markov chain in this order (denoted as $Y-X-M$ ) if registers $Y$ and $M$ are independent given $X$. Hayden, Josza, Petz, and Winter [14] showed that an analogous property holds for quantum Markov states. In particular, they showed that a state $\sigma^{R B C} \in \mathrm{D}\left(\mathcal{H}^{R} \otimes\right.$ $\mathcal{H}^{B} \otimes \mathcal{H}^{C}$ ) is a Markov state if and only if there is a decomposition of the space $\mathcal{H}^{B}$ into a direct sum of tensor products as

$$
\mathcal{H}^{B}=\bigoplus_{j} \mathcal{H}^{B_{j}^{R}} \otimes \mathcal{H}^{B_{j}^{C}}
$$

such that

$$
\sigma^{R B C}=\bigoplus_{j} p(j) \sigma_{j}^{R B_{j}^{R}} \otimes \sigma_{j}^{B_{j}^{C} C}
$$

where $\sigma_{j}^{R B_{j}^{R}} \in \mathrm{D}\left(\mathcal{H}^{R} \otimes \mathcal{H}^{B_{j}^{R}}\right), \sigma_{j}^{B_{j}^{C} C} \in \mathrm{D}\left(\mathcal{H}^{B_{j}^{C}} \otimes \mathcal{H}^{C}\right)$ and $p$ is a probability distribution.

For a state $\psi^{R B C}$, we say that $\sigma^{R B C}$ is a Markov extension of $\psi^{R B}$ if $\sigma^{R B}=\psi^{R B}$ and $\sigma^{R B C}$ is a Markov state. We denote the set of all Markov extensions of $\psi^{R B}$ by $\mathrm{QMC}_{R-B-C}^{\psi}$. Note that $\mathrm{QMC}_{R-B-C}^{\psi}$ is non-empty, as we may take $\sigma^{R B C}:=\psi^{R B} \otimes \psi^{C}$. The following lemma relates the quantum conditional mutual information to quantum Markov extensions.

Lemma 2.8. For any tripartite quantum state $\psi^{R B C}$, and any quantum Markov extension $\sigma^{R B C} \in$ $\mathrm{QMC}_{R-B-C}^{\psi}$, it holds that

$$
\mathrm{I}(R: C \mid B)_{\psi}=\mathrm{D}\left(\psi^{R B C} \| \sigma^{R B C}\right)-\mathrm{D}\left(\psi^{B C} \| \sigma^{B C}\right) .
$$

The proof of this lemma is implicit in Ref. [10, Lemma 1], but we provide a proof here for completeness.

Proof: For sake of clarity, in this proof, we suppress tensor products with the identity in expressions involving sums or products of quantum states over different sequences of registers. For example, we write $\omega^{X Y}+\tau^{Y Z}$ to represent the sum $\omega^{X Y} \otimes \mathbb{1}^{Z}+\mathbb{1}^{X} \otimes \tau^{Y Z}$, and $\omega^{X Y} \tau^{Y Z}$ to represent the product $\left(\omega^{X Y} \otimes \mathbb{1}^{Z}\right)\left(\mathbb{1}^{X} \otimes \tau^{Y Z}\right)$. All the expressions involving entropy and mutual information are with respect to the state $\psi$.

Consider any quantum Markov chain $\sigma^{R B C}$ satisfying $\sigma^{R B}=\psi^{R B}$. From Eq. (2.5), we have

$$
\log \sigma^{R B C}=\bigoplus_{j}\left(\log \left(p(j) \sigma_{j}^{R B_{j}^{R}}\right)+\log \sigma_{j}^{B_{j}^{C} C}\right)
$$

and similarly,

$$
\log \sigma^{B C}=\bigoplus_{j}\left(\log \left(p(j) \sigma_{j}^{B_{j}^{R}}\right)+\log \sigma_{j}^{B_{j}^{C} C}\right)
$$


Thus, we can evaluate

$$
\begin{aligned}
\mathrm{D}\left(\psi^{R B C} \| \sigma^{R B C}\right)-\mathrm{D}\left(\psi^{B C} \| \sigma^{B C}\right) \\
=\operatorname{Tr}\left(\psi^{R B C} \log \psi^{R B C}\right)-\operatorname{Tr}\left(\psi^{R B C} \log \sigma^{R B C}\right)-\operatorname{Tr}\left(\psi^{B C} \log \psi^{B C}\right)+\operatorname{Tr}\left(\psi^{B C} \log \sigma^{B C}\right) \\
=\operatorname{S}(B C)-\mathrm{S}(R B C)-\sum_{j} \operatorname{Tr}\left(\psi^{R B C} \log \left(p(j) \sigma_{j}^{R B_{j}^{R}}\right)\right)-\sum_{j} \operatorname{Tr}\left(\psi^{R B C} \log \sigma_{j}^{B_{j}^{C} C}\right) \\
\quad+\sum_{j} \operatorname{Tr}\left(\psi^{B C} \log \left(p(j) \sigma_{j}^{B_{j}^{R}}\right)\right)+\sum_{j} \operatorname{Tr}\left(\psi^{B C} \log \sigma_{j}^{B_{j}^{C} C}\right) .
\end{aligned}
$$

Since $\operatorname{Tr}\left(\psi^{R B C} \log \sigma_{j}^{B_{j}^{C} C}\right)=\operatorname{Tr}\left(\psi^{B C} \log \sigma_{j}^{B_{j}^{C} C}\right)$, the above equation can be simplified to obtain

$$
\begin{aligned}
\mathrm{D}\left(\psi^{R B C} \| \sigma^{R B C}\right)-\mathrm{D}\left(\psi^{B C} \| \sigma^{B C}\right) \\
=\mathrm{S}(B C)-\mathrm{S}(R B C)-\sum_{j} \operatorname{Tr}\left(\psi^{R B C} \log \left(p(j) \sigma_{j}^{R B_{j}^{R}}\right)\right)+\sum_{j} \operatorname{Tr}\left(\psi^{B C} \log \left(p(j) \sigma_{j}^{B_{j}^{R}}\right)\right) \\
=\mathrm{S}(B C)-\mathrm{S}(R B C)-\operatorname{Tr}\left(\psi^{R B C} \log \left(\bigoplus_{j} p(j) \sigma_{j}^{R B_{j}^{R}}\right)\right)+\operatorname{Tr}\left(\psi^{B C} \log \left(\bigoplus_{j} p(j) \sigma_{j}^{B_{j}^{R}}\right)\right) \\
=\mathrm{S}(B C)-\mathrm{S}(R B C)-\operatorname{Tr}\left(\psi^{R B C} \log \bigoplus_{j}\left(p(j) \sigma_{j}^{R B_{j}^{R}} \otimes \sigma_{j}^{B_{j}^{C}}\right)\right) \\
\quad+\operatorname{Tr}\left(\psi^{B C} \log \bigoplus_{j}\left(p(j) \sigma_{j}^{B_{j}^{R}} \otimes \sigma_{j}^{B_{j}^{C}}\right)\right)
\end{aligned}
$$

where the last equality above follows by noting that

$$
\operatorname{Tr}\left(\psi^{R B C} \log \sigma_{j}^{B_{j}^{C}}\right)=\operatorname{Tr}\left(\psi^{B C} \log \sigma_{j}^{B_{j}^{C}}\right) .
$$

Since $\psi^{R B}=\sigma^{R B}$, we get that

$$
\begin{aligned}
\mathrm{D}\left(\psi^{R B C} \| \sigma^{R B C}\right)-\mathrm{D}\left(\psi^{B C} \| \sigma^{B C}\right) & =\mathrm{S}(B C)-\mathrm{S}(R B C)-\operatorname{Tr}\left(\psi^{R B C} \log \sigma^{R B}\right)+\operatorname{Tr}\left(\psi^{B C} \log \sigma^{B}\right) \\
& =\mathrm{S}(B C)-\mathrm{S}(R B C)-\operatorname{Tr}\left(\psi^{R B} \log \psi^{R B}\right)+\operatorname{Tr}\left(\psi^{B} \log \psi^{B}\right) \\
& =\mathrm{S}(B C)-\mathrm{S}(R B C)+\mathrm{S}(R B)-\mathrm{S}(B) \\
& =\mathrm{I}(R: C \mid B) .
\end{aligned}
$$

This completes the proof.

For a Markov extension $\sigma \in \mathrm{QMC}_{R-B-C}^{\psi}$, let $\Pi_{j}^{\sigma}$ be the orthogonal projection operator onto the $j$-th subspace of the register $B$ given by the decomposition corresponding to the Markov state $\sigma$ as described above. In other words, $\Pi_{j}^{\sigma}$ is the projection onto the Hilbert space $\mathcal{H}^{B_{j}^{R}} \otimes \mathcal{H}^{B_{j}^{C}}$ in Eq. (2.4). For a quantum state $\psi^{R B C}$, we define

$$
\mathrm{ME}_{R-B-C}^{\epsilon, \psi}:=\left\{\sigma \in \mathrm{QMC}_{R-B-C}^{\psi} \mid \text { for all } j, \sigma_{j}^{B_{j}^{C} C} \in \mathrm{B}^{\epsilon}\left(\operatorname{Tr}_{B_{j}^{R}}\left[\left(\Pi_{j}^{\sigma} \otimes \mathbb{1}\right) \psi^{B C}\left(\Pi_{j}^{\sigma} \otimes \mathbb{1}\right)\right]\right)\right\} \text {. }
$$

Informally, this is the subset of Markov extensions $\sigma$ of $\psi$ such that the restrictions of $\sigma$ and $\psi$ to the $j$-th subspace in the decomposition of $\sigma$ agree well on the registers $B_{j}^{C} C$. Again, the state $\sigma^{R B C}:=\psi^{R B} \otimes \psi^{C}$ belongs to $\mathrm{ME}_{R-B-C}^{\epsilon, \psi}$ for every $\epsilon \geq 0$, so the set is non-empty. 


\subsection{Quantum state redistribution}

Consider a pure state $|\psi\rangle^{R A B C}$ shared between Referee $(R)$, Alice $(A C)$ and Bob $(B)$. In an $\epsilon$-error quantum state redistribution protocol, Alice and Bob share an entangled state $|\theta\rangle^{E_{A} E_{B}}$, register $E_{A}$ with Alice and register $E_{B}$ with Bob. Alice applies an encoding operation $\mathcal{E}: \mathrm{L}\left(\mathcal{H}^{A C E_{A}}\right) \rightarrow \mathrm{L}\left(\mathcal{H}^{A Q}\right)$, and sends the register $Q$ to Bob. Then, Bob applies a decoding operation $\mathcal{D}: \mathrm{L}\left(\mathcal{H}^{Q B E_{B}}\right) \rightarrow \mathrm{L}\left(\mathcal{H}^{B C}\right)$. The output of the protocol is the state $\phi^{R A B C}$ with the property that $\mathrm{P}\left(\psi^{R A B C}, \phi^{R A B C}\right) \leq \epsilon$, and the communication cost of the protocol is $\log |Q|$.

To derive the upper bound in Theorem 1.1, we use an existing protocol due to Anshu, Jain and Warsi [7] which we call the AJW protocol in the sequel. The bound is derived by combining the AJW protocol with a decoupling technique via embezzling described in Section 2.4.

\subsubsection{AJW protocol}

The AJW protocol is based on the techniques of convex-split lemma (introduced in Ref. [2]) and position-based decoding (introduced in Ref. [6]).

Let $n$ be an integer, $\rho^{A B} \in \mathrm{D}\left(\mathcal{H}^{A B}\right)$ and $\sigma^{B} \in \mathrm{D}\left(\mathcal{H}^{B}\right)$. Consider the quantum state $\tau^{A B_{1} \ldots B_{n}}$ derived by adding $n-1$ independent copies of $\sigma^{B}$ in tensor product with $\rho^{A B}$ and swapping $i$-th copy of $\sigma^{B}$ with $\rho^{B}$ for uniformly random $i \in[n-1]$. The convex-split lemma states that the state $\tau^{A B_{1} \ldots B_{n}}$ is almost indistinguishable from the product state $\rho^{A} \otimes\left(\sigma^{B}\right)^{\otimes n}$, provided that $n$ is large enough.

Lemma 2.9 (Convex-Split [2]). Let $\rho^{A B} \in \mathrm{D}\left(\mathcal{H}^{A B}\right)$ and $\sigma^{B} \in \mathrm{D}\left(\mathcal{H}^{B}\right)$ be quantum states with $\mathrm{D}_{\max }\left(\rho^{A B} \| \rho^{A} \otimes \sigma^{B}\right)=k$ for some finite number $k$. Let $\delta>0$ and $n=\left\lceil\frac{2^{k}}{\delta}\right\rceil$. Define the following state

$$
\tau^{A B_{1} B_{2} \ldots B_{n}} \quad:=\frac{1}{n} \sum_{j=1}^{n} \rho^{A B_{j}} \otimes \sigma^{B_{1}} \otimes \cdots \otimes \sigma^{B_{j-1}} \otimes \sigma^{B_{j+1}} \otimes \cdots \otimes \sigma^{B_{n}},
$$

on $n+1$ registers $A, B_{1}, B_{2}, \ldots, B_{n}$, where for all $i \in[n], \rho^{A B_{i}}=\rho^{A B}$ and $\sigma^{B_{i}}=\sigma^{B}$. Then, we have

$$
\mathrm{P}\left(\tau^{A B_{1} \ldots B_{n}}, \tau^{A} \otimes \sigma^{B_{1}} \otimes \cdots \otimes \sigma^{B_{n}}\right) \leq \sqrt{\delta} .
$$

The above lemma provides the conditions under which the correlation in between registers $A$ and $B$ in $\rho$ is hidden by a certain convex combination of quantum states. A dual problem is to find conditions sufficient for identifying the location of the desired correlation in a convex combination. This task is achievable via position-based decoding technique using quantum hypothesis testing.

Lemma 2.10 (Position-based decoding [6]). Let $\epsilon>0$, and $\rho^{A B} \in \mathrm{D}\left(\mathcal{H}^{A B}\right)$ and $\sigma^{B} \in \mathrm{D}\left(\mathcal{H}^{B}\right)$ be quantum states such that $\operatorname{supp}\left(\rho^{B}\right) \subseteq \operatorname{supp}\left(\sigma^{B}\right)$. Let $n:=\left\lceil\epsilon 2^{\mathrm{D}_{\mathrm{H}}^{\epsilon}\left(\rho^{A B} \| \rho^{A} \otimes \sigma^{B}\right)}\right]$, and for every $j \in[n]$,

$$
\tau_{j}^{A B_{1} \ldots B_{n}} \quad:=\rho^{A B_{j}} \otimes \sigma^{B_{1}} \otimes \cdots \otimes \sigma^{B_{j-1}} \otimes \sigma^{B_{j+1}} \otimes \cdots \otimes \sigma^{B_{n}} .
$$

There exists a set of POVM operators $\left\{\Lambda_{j}: j \in[n+1]\right\}$ on registers $A B_{1} B_{2} \ldots B_{n}$ such that

$$
\sum_{j=1}^{n+1} \Lambda_{j}=\mathbb{1}
$$


and for all $j \in[n]$,

$$
\operatorname{Tr}\left[\Lambda_{j} \tau_{j}^{A B_{1} \ldots B_{n}}\right] \geq 1-6 \epsilon
$$

The above statement is slightly different from the one in Ref. [6] because of a minor difference in defining quantum hypothesis testing relative entropy.

Let $|\psi\rangle^{R A B C}$ be the quantum state shared between Alice, Bob and Referee where registers $(A C)$ are with Alice, register $B$ is with Bob and register $R$ is with Referee, and $\psi^{\prime R B C} \in \mathrm{B}^{\epsilon}\left(\psi^{R B C}\right)$. The AJW protocol works as follows.

AJW protocol: Alice and Bob initially share $m=\left\lceil 2^{\beta} / \epsilon^{2}\right\rceil$ copies of a purification $|\sigma\rangle^{L C}$ of $\sigma^{C}$ where $\beta=\mathrm{D}_{\max }\left(\psi^{\prime R B C} \| \psi^{\prime R B} \otimes \sigma^{C}\right)$. Their global state is $|\psi\rangle^{R A B C} \otimes|\sigma\rangle^{L_{1} C_{1}} \otimes \ldots \otimes|\sigma\rangle^{L_{m} C_{m}}$. Let $b$ be the smallest integer such that $\log b \geq \mathrm{D}_{\mathrm{H}}^{\epsilon^{2}}\left(\psi^{\prime B C} \| \psi^{\prime B} \otimes \sigma^{C}\right)-\log \frac{1}{\epsilon^{2}}$. By performing a proper unitary operator, Alice transforms the global state into a state close to the state

$\frac{1}{m} \sum_{j=1}^{m}|\lfloor j / b\rfloor\rangle^{J_{1}}|j(\bmod b)\rangle^{J_{2}}|0\rangle^{L_{j}}|\psi\rangle^{R A B C_{j}} \otimes|\sigma\rangle^{L_{1} C_{1}} \otimes \ldots \otimes|\sigma\rangle^{L_{j-1} C_{j-1}} \otimes|\sigma\rangle^{L_{j+1} C_{j+1}} \otimes \ldots \otimes|\sigma\rangle^{L_{n} C_{n}}$.

This is possible due to the Uhlmann theorem, the convex-split lemma and the choice of $m$. Alice sends register $J_{1}$ to Bob with communication cost at most $1 / 2(\log m-\log b)$ using superdense coding. Then, for each $j_{2} \leq b$, Bob swaps registers $C_{j_{2}}$ and $C_{j_{2}+b j_{1}}$, conditioned on $J_{1}=j_{1}$. At this point, registers $R B C_{1} \ldots C_{b}$ are in a state close to

$$
\frac{1}{b} \sum_{j_{2}=1}^{b} \psi^{R B C_{j_{2}}} \otimes \sigma^{C_{1}} \otimes \ldots \otimes \sigma^{C_{j_{2}-1}} \otimes \sigma^{C_{j_{2}+1}} \otimes \ldots \otimes \sigma^{C_{b}}
$$

Then, Bob uses position-based decoding to determine the index $j_{2}$ for which register $C_{j_{2}}$ is correlated with registers $R B$. This is possible by the choice of $b$. Since the state over registers $R B C_{j_{2}}$ is close to $\psi^{R B C}$ and it is independent of the state over registers $C_{1} \ldots C_{j_{2}-1}, C_{j_{2}+1}, \ldots, C_{b}$, the register purifying registers $R B C_{j_{2}}$ is with Alice and she can transform it to the register $A$ such that the final state over registers $R A B C_{j_{2}}$ is close to $\psi^{R A B C}$.

The following theorem states the communication cost and the error in the final state of the above protocol.

Theorem 2.11 ([7]). Let $\epsilon \in(0,1)$, and $|\psi\rangle^{R A B C}$ be a pure quantum state shared between Referee $(R)$, Alice $(A C)$ and $B o b(B)$. There exists an entanglement-assisted one-way protocol operated by Alice and Bob which starts in the state $|\psi\rangle^{R A B C}$, and outputs a state $\phi^{R A B C} \in \mathrm{B}^{9 \epsilon}\left(\psi^{R A B C}\right)$, and the number of qubits communicated by Alice and Bob is upper bounded by

$$
\frac{1}{2} \inf _{\sigma^{C}} \inf _{\psi^{\prime} \in \mathrm{B}^{\epsilon}\left(\psi^{R B C}\right)}\left(\mathrm{D}_{\max }\left(\psi^{\prime R B C} \| \psi^{\prime R B} \otimes \sigma^{C}\right)-\mathrm{D}_{\mathrm{H}}^{\epsilon^{2}}\left(\psi^{\prime B C} \| \psi^{\prime B} \otimes \sigma^{C}\right)\right)+\log \frac{1}{\epsilon^{2}} .
$$

For a complete proof (including correctness and error analysis), see the proof of Theorem 1 in Ref. [7. 


\subsection{Decoupling classical-quantum states via embezzlement}

Embezzlement refers to a process introduced by van Dam and Hayden [27] in which any bipartite quantum state, possibly entangled, can be produced from a bipartite catalyst, called the embezzling quantum state, using only local unitary operations. For an integer $n$ and registers $D$ and $D^{\prime}$ with $|D|=\left|D^{\prime}\right| \geq n$, the embezzling state is defined as

$$
|\xi\rangle^{D D^{\prime}} \quad:=\frac{1}{\sqrt{S(n)}} \sum_{i=1}^{n} \frac{1}{\sqrt{i}}|i\rangle^{D}|i\rangle^{D^{\prime}},
$$

where $S(n):=\sum_{i=1}^{n} \frac{1}{i}$. Van Dam and Hayden showed that for every bipartite state $|\phi\rangle^{A B}$ with Schmidt rank $m$, there exists local isometries $V_{A}: \mathcal{H}^{D} \rightarrow \mathcal{H}^{D A}$ and $V_{B}: \mathcal{H}^{D^{\prime}} \rightarrow \mathcal{H}^{D^{\prime} B}$ such that

$$
\mathrm{P}\left(\left(V_{A} \otimes V_{B}\right)|\xi\rangle,|\xi\rangle \otimes|\phi\rangle\right) \leq \delta,
$$

provided that $n \geq m^{2 / \delta^{2}}$. Therefore, by using $n$ large enough, arbitrarily good accuracy in embezzlement can be achieved.

For a fixed $a \leq n$, a close variant of the above embezzling state is defined as

$$
\left|\xi_{a: n}\right\rangle^{D D^{\prime}} \quad:=\frac{1}{\sqrt{S(a, n)}} \sum_{i=a}^{n} \frac{1}{\sqrt{i}}|i\rangle^{D}|i\rangle^{D^{\prime}} .
$$

Using these states, Lemma 2.12 achieves the embezzling of the uniform distribution where the closeness is guaranteed in max-relative entropy.

Lemma $2.12([3])$. Let $\delta \in\left(0, \frac{1}{15}\right)$, and $a, b, n \in \mathbb{Z}$ be positive integers such that $a \geq b$ and $n \geq a^{1 / \delta}$. Let $D$ and $E$ be registers with $|D| \geq n$ and $|E| \geq b$. Let $W_{b}$ be a unitary operation that acts as

$$
W_{b}|i\rangle^{D}|0\rangle^{E} \quad=|\lfloor i / b\rfloor\rangle^{D}|i(\bmod b)\rangle^{E} \quad \forall i \in\{0, \ldots|D|-1\},
$$

and $\Pi_{b} \in \operatorname{Pos}\left(\mathcal{H}^{D E}\right)$ be the projection operator onto the support of $W_{b}\left(\xi_{a: n}^{D} \otimes|0\rangle\left\langle\left. 0\right|^{E}\right) W_{b}^{\dagger}\right.$. It holds that

$$
W_{b}\left(\xi_{a: n}^{D} \otimes|0\rangle\left\langle\left. 0\right|^{E}\right) W_{b}^{\dagger} \preceq(1+15 \delta) \xi_{1: n}^{D} \otimes \mu_{b}^{E}\right.
$$

and

$$
\Pi_{b}\left(\xi_{1: n}^{D} \otimes \mu_{b}^{E}\right) \Pi_{b} \preceq 2 \cdot W_{b}\left(\xi_{a: n}^{D} \otimes|0\rangle\left\langle\left. 0\right|^{E}\right) W_{b}^{\dagger} .\right.
$$

where $\mu_{b}^{E}=\frac{1}{b} \sum_{e=0}^{b-1}|e\rangle\langle e|$.

The proof of Eq. (2.14) is due to Anshu and Jain [3, Claim 1], and Eq. (2.15) follows from a similar argument. For completeness, we provide a proof for Lemma 2.12 below. 
Proof: Let $W_{b}$ be a unitary operator satisfying Eq. (2.13). We have

$$
\begin{aligned}
W_{b}\left(\xi_{a: n}^{D} \otimes|0\rangle\left\langle\left. 0\right|^{E}\right) W_{b}^{\dagger}\right. & =\frac{1}{S(a, n)} \sum_{i=1}^{n} \frac{1}{i} W_{b}\left(|i\rangle\left\langle\left. i\right|^{D} \otimes \mid 0\right\rangle\left\langle\left. 0\right|^{E}\right) W_{b}^{\dagger}\right. \\
& =\left.\frac{1}{S(a, n)} \sum_{i=1}^{n} \frac{1}{i}|\lfloor i / b\rfloor\rangle\langle i / b\rfloor\right|^{D} \otimes|i(\bmod b)\rangle\left\langle\left. i(\bmod b)\right|^{E}\right. \\
& =\frac{1}{S(a, n)} \sum_{i^{\prime}=\left\lfloor\frac{a}{b}\right\rfloor}^{\left\lfloor\frac{n}{b}\right\rfloor} \sum_{e=0}^{\min \left\{b-1, n-i^{\prime} b\right\}} \frac{1}{b i^{\prime}+e}\left|i^{\prime}\right\rangle\left\langle\left. i^{\prime}\right|^{D} \otimes \mid e\right\rangle\left\langle\left. e\right|^{E}\right. \\
& \preceq \frac{1}{S(a, n)} \sum_{i^{\prime}=\left\lfloor\frac{a}{b}\right\rfloor}^{\left\lfloor\frac{b}{b}\right\rfloor} \sum_{e=0}^{b-1} \frac{1}{b i^{\prime}}\left|i^{\prime}\right\rangle\left\langle\left. i^{\prime}\right|^{D} \otimes \mid e\right\rangle\left\langle\left. e\right|^{E}\right. \\
& \preceq \frac{S(1, n)}{S(a, n)} \xi_{1: n}^{D} \otimes \mu_{b}^{E} .
\end{aligned}
$$

In Ref. [18, it is shown that $\left|S(a, n)-\log \frac{n}{a}\right| \leq 4$. Since $n \geq a^{1 / \delta}$, we have

$$
\frac{S(1, n)}{S(a, n)} \leq \frac{\log n+4}{\log n-\log a-4} \leq \frac{1+4 \delta}{1-5 \delta} \leq 1+15 \delta .
$$

Now, Eq. (2.17) and Eq. (2.18) together imply Eq. (2.14). It remains to prove Eq. (2.15). Let $\Pi_{b} \in$ $\operatorname{Pos}\left(\mathcal{H}^{D E}\right)$ be the projection operator onto the support of $W_{b}\left(\xi_{a: n}^{D} \otimes|0\rangle\left\langle\left. 0\right|^{E}\right) W_{b}^{\dagger}\right.$. Eq. (2.16) implies that

$$
\Pi_{b}=\sum_{i^{\prime}=\left\lfloor\frac{a}{b}\right\rfloor}^{\left\lfloor\frac{n}{b}\right\rfloor} \sum_{e=0}^{\min \left\{b-1, n-i^{\prime} b\right\}}\left|i^{\prime}\right\rangle\left\langle\left. i^{\prime}\right|^{D} \otimes \mid e\right\rangle\left\langle\left. e\right|^{E} .\right.
$$

Thus,

$$
\begin{aligned}
\Pi_{b}\left(\xi_{1: n}^{D} \otimes \mu_{b}^{E}\right) \Pi_{b} & =\frac{1}{S(1, n)} \sum_{i^{\prime}=\left\lfloor\frac{a}{b}\right\rfloor}^{\left\lfloor\frac{n}{b}\right\rfloor} \sum_{e=0}^{\min \left\{b-1, n-i^{\prime} b\right\}} \frac{1}{b i^{\prime}}\left|i^{\prime}\right\rangle\left\langle\left. i^{\prime}\right|^{D} \otimes \mid e\right\rangle\left\langle\left. e\right|^{E}\right. \\
& \preceq \frac{1}{S(1, n)} \sum_{i^{\prime}=\left\lfloor\frac{a}{b}\right\rfloor}^{\left\lfloor\frac{n}{b}\right\rfloor} \sum_{e=0}^{\min \left\{b-1, n-i^{\prime} b\right\}} \frac{2}{b i^{\prime}+e}\left|i^{\prime}\right\rangle\left\langle\left. i^{\prime}\right|^{D} \otimes \mid e\right\rangle\left\langle\left. e\right|^{E}\right. \\
& =\frac{2 \cdot S(a, n)}{S(1, n)} W_{b}\left(\xi_{a: n}^{D} \otimes|0\rangle\left\langle\left. 0\right|^{E}\right) W_{b}^{\dagger} \quad\right. \text { (by Eq. (2.14) ) } \\
& \preceq 2 \cdot W_{b}\left(\xi_{a: n}^{D} \otimes|0\rangle\left\langle\left. 0\right|^{E}\right) W_{b}^{\dagger},\right.
\end{aligned}
$$

where the first inequality holds since $b i^{\prime}+e \leq 2 b i^{\prime}$ for $i^{\prime} \geq 1$ and $0 \leq e \leq b-1$, and the second inequality holds since $S(a, n) \leq S(1, n)$.

As a corollary of the above lemma, Anshu and Jain [3] showed that the embezzling state $\xi_{a: n}^{D}$ can be used almost catalytically to flatten any quantum state using unitary operations.

Corollary 2.13 ([3], Eq. (6)). Let $\rho \in \mathrm{D}\left(\mathcal{H}^{C}\right)$ be a quantum state with spectral decomposition $\rho^{C}=$ $\sum_{c} q(c)\left|v_{c}\right\rangle\left\langle\left. v_{c}\right|^{C}\right.$. Let $\delta \in\left(0, \frac{1}{15}\right)$ and $\gamma \in(0,1)$ such that $\frac{|C|}{\gamma}$ is an integer and all eigenvalues $q(c)$ 
are integer multiples of $\frac{\gamma}{|C|}$. Let $a:=\frac{|C|}{\gamma} \max _{c} q(c), n=a^{1 / \delta}$, and $D$ and $E$ be quantum registers with $|D| \geq n$ and $|E|=a$. Let $W \in \mathrm{U}\left(\mathcal{H}^{C E D}\right)$ be the unitary operator defined as

$$
W \quad:=\sum_{c}\left|v_{c}\right\rangle\left\langle\left. v_{c}\right|^{C} \otimes W_{b(c)}\right.
$$

and $\Pi \in \operatorname{Pos}\left(\mathcal{H}^{C E D}\right)$ be the projection operator defined as

$$
\Pi \quad:=\sum_{c}\left|v_{c}\right\rangle\left\langle\left. v_{c}\right|^{C} \otimes \Pi_{b(c)},\right.
$$

where $W_{b(c)}$ and $\Pi_{b(c)}$ are the operators defined in Lemma 2.12 with $b(c):=\frac{q(c)|C|}{\gamma}$. Then, we have

$$
W\left(\rho^{C} \otimes|0\rangle\left\langle\left. 0\right|^{E} \otimes \xi_{a: n}^{D}\right) W^{\dagger} \preceq \quad(1+15 \delta) \rho^{C E} \otimes \xi_{1: n}^{D}\right.
$$

and

$$
\Pi\left(\rho^{C E} \otimes \xi_{1: n}^{D}\right) \Pi \preceq 2 \cdot W\left(\rho^{C} \otimes|0\rangle\left\langle\left. 0\right|^{E} \otimes \xi_{a: n}^{D}\right) W^{\dagger},\right.
$$

where $\rho^{C E}:=\frac{\gamma}{|C|} \sum_{c}\left|v_{c}\right\rangle\left\langle\left. v_{c}\right|^{C} \otimes \sum_{e=0}^{b(c)-1} \mid e\right\rangle\left\langle\left. e\right|^{E}\right.$ is an extension of $\rho^{C}$ with flat spectrum.

The proof of Eq. (2.19) is provided in Ref. [3, Eq. (6)], and Eq. (2.20) follows from a Eq. (2.15). For completeness, we provide a proof for Corollary 2.13 below.

Proof: Let $W$ be the unitary operator defined in the statement of the corollary . We have

$$
\begin{aligned}
& W\left(\rho^{C} \otimes|0\rangle\left\langle\left. 0\right|^{E} \otimes \xi_{a: n}^{D}\right) W^{\dagger}\right. \\
& =\sum_{c} q(c)\left|v_{c}\right\rangle\left\langlev _ { c } | ^ { C } \otimes W _ { b ( c ) } \left(|0\rangle\left\langle\left. 0\right|^{E} \otimes \xi_{a: n}^{D}\right) W_{b(c)}^{\dagger}\right.\right. \\
& \preceq \quad(1+15 \delta) \sum_{c} q(c)\left|v_{c}\right\rangle\left\langle\left. v_{c}\right|^{C} \otimes \frac{\gamma}{q(c)|C|} \sum_{e=0}^{b(c)-1} \mid e\right\rangle\left\langle\left. e\right|^{E} \otimes \xi_{a: n}^{D}\right. \\
& =(1+15 \delta) \rho^{C E} \otimes \xi_{a: n}^{D},
\end{aligned}
$$

where the inequality follows from Lemma 2.12, So, it remains to prove Eq. (2.20). Let $\Pi$ be the projection operator defined in the statement of the corollary. We have

$$
\begin{aligned}
\Pi\left(\rho^{C E} \otimes \xi_{1: n}^{D}\right) \Pi & =\frac{\gamma}{|C|} \sum_{c} b(c)\left|v_{c}\right\rangle\left\langle\left. v_{c}\right|^{C} \otimes \Pi_{b(c)}\left(\mu_{b(c)}^{E} \otimes \xi_{a: n}^{D}\right) \Pi_{b(c)}\right. \\
& \preceq 2 \sum_{c} q(c)\left|v_{c}\right\rangle\left\langlev _ { c } | ^ { C } \otimes W _ { b ( c ) } \left(|0\rangle\left\langle\left. 0\right|^{E} \otimes \xi_{a: n}^{D}\right) W_{b(c)}^{\dagger}\right.\right. \\
& =2 \cdot W\left(\rho^{C} \otimes|0\rangle\left\langle\left. 0\right|^{E} \otimes \xi_{a: n}^{D}\right) W^{\dagger}\right.
\end{aligned}
$$

where the inequality is a consequence of Lemma 2.12 ,

We use the above flattening procedure to decouple the quantum register in a classical-quantum state. 
Corollary 2.14. Consider a classical-quantum state $\rho^{J C}=\sum_{j} p(j)|j\rangle\left\langle\left. j\right|^{J} \otimes \rho_{j}^{C}\right.$, where $p$ is a probability distribution and $\rho_{j}^{C} \in \mathrm{D}\left(\mathcal{H}^{C}\right)$. Let $\delta \in\left(0, \frac{1}{15}\right)$ and $\gamma \in(0,1)$ such that $a:=\frac{|C|}{\gamma}$ is an integer and eigenvalues of all $\rho_{j}^{C}$ are integer multiples of $\frac{\gamma}{|C|}$. Let $n=a^{1 / \delta}, D$ and $E$ be quantum registers with $|D| \geq n$ and $|E|=a$. Then, there exists a unitary operator $U \in \mathrm{U}\left(\mathcal{H}^{J C E D}\right)$, read-only on register $J$, and a projection operator $\widetilde{\Pi} \in \operatorname{Pos}\left(\mathcal{H}^{J C E D}\right)$ such that

$$
\begin{aligned}
& U\left(\rho^{J C} \otimes|0\rangle\left\langle\left. 0\right|^{E} \otimes \xi_{a: n}^{D}\right) U^{\dagger} \preceq(1+15 \delta) \rho^{J} \otimes \nu^{C E} \otimes \xi_{1: n}^{D},\right. \\
& \widetilde{\Pi}\left(\rho^{J} \otimes \nu^{C E} \otimes \xi_{1: n}^{D}\right) \widetilde{\Pi} \preceq 2 \cdot U\left(\rho^{J C} \otimes|0\rangle\left\langle\left. 0\right|^{E} \otimes \xi_{a: n}^{D}\right) U^{\dagger},\right.
\end{aligned}
$$

and

$$
\operatorname{Tr}\left[\widetilde{\Pi} U\left(\rho^{J C} \otimes|0\rangle\left\langle\left. 0\right|^{E} \otimes \xi_{a: n}^{D}\right) U^{\dagger}\right]=1,\right.
$$

where $\nu^{C E}=\frac{1}{a} \sum_{s=0}^{a-1}|s\rangle\left\langle\left. s\right|^{C E}\right.$.

Proof: Notice that integers $a$ and $n$ and registers $D$ and $E$ satisfy required properties in Corollary 2.13. For each $j$, let $W^{(j)}$ be the unitary operator given by Corollary 2.13 for flattening $\rho_{j}^{C}=\sum_{c} q_{j}(c)\left|v_{j, c}\right\rangle\left\langle v_{j, c}\right|$. Hence, we can flatten all $\rho_{j}^{C}$ simultaneously using the unitary operator $U_{1}=\sum_{j}|j\rangle\langle j| \otimes W^{(j)}$, and we get

$$
U_{1}\left(\rho ^ { J C } \otimes | 0 \rangle \langle 0 | ^ { E } \otimes \xi _ { a : n } ^ { D } ) U _ { 1 } ^ { \dagger } \preceq \quad ( 1 + 1 5 \delta ) \sum _ { j } p ( j ) | j \rangle \left\langle\left.j\right|^{J} \otimes \rho_{j}^{C E} \otimes \xi_{1: n}^{D},\right.\right.
$$

where $\rho_{j}^{C E}=\frac{\gamma}{|C|} \sum_{c}\left|v_{j, c}\right\rangle\left\langle\left. v_{j, c}\right|^{C} \otimes \sum_{e=0}^{q_{j}(c)|C| / \gamma} \mid e\right\rangle\left\langle\left. e\right|^{E}\right.$ is an extension of $\rho^{C}$ with flat spectrum. For each $j$, the support of $\rho_{j}^{C}$ has dimension $\sum_{c} q_{j}(c) \frac{|C|}{\gamma}=a$, which is independent of $j$. Hence, there exists a unitary operator $V^{(j)}$ mapping $\rho_{j}^{C E}$ to $\nu^{C E}$. Let $U_{2} \in \mathrm{U}\left(\mathcal{H}^{J C E}\right)$ be the unitary operator $U_{2}:=\sum_{j}|j\rangle\langle j| \otimes V^{(j)}$. Then, the unitary operator $U:=U_{2} U_{1}$ satisfies Eq. (2.21).

Now, for each $j$, let $\Pi^{(j)} \in \operatorname{Pos}\left(\mathcal{H}^{C E D}\right)$ be the projection operator given by Corollary 2.13, Define $\Pi^{\prime}:=\sum_{j}|j\rangle\langle j| \otimes \Pi^{(j)}$ and $\widetilde{\Pi}:=U_{2} \Pi^{\prime} U_{2}^{\dagger}$. We have

$$
\begin{aligned}
\widetilde{\Pi}\left(\rho^{J} \otimes \nu^{C E} \otimes \xi_{1: n}^{D}\right) \widetilde{\Pi} & =U_{2} \Pi^{\prime} U_{2}^{\dagger}\left(\rho^{J} \otimes \nu^{C E} \otimes \xi_{1: n}^{D}\right) U_{2} \Pi^{\prime} U_{2}^{\dagger} \\
& =U_{2} \Pi^{\prime}\left(\sum_{j} p(j)|j\rangle\left\langle\left. j\right|^{J} \otimes \rho_{j}^{C E} \otimes \xi_{1: n}^{D}\right) \Pi^{\prime} U_{2}^{\dagger}\right. \\
& =U_{2}\left(\sum_{j} p(j)|j\rangle\left\langle\left. j\right|^{J} \otimes \Pi^{(j)}\left(\rho_{j}^{C E} \otimes \xi_{1: n}^{D}\right) \Pi^{(j)}\right) U_{2}^{\dagger}\right. \\
& \preceq 2 \cdot U_{2}\left(\sum _ { j } p ( j ) | j \rangle \left\langle\left.j\right|^{J} \otimes W^{(j)}\left(\rho_{j}^{C} \otimes|0\rangle\left\langle\left. 0\right|^{E} \otimes \xi_{a: n}^{D}\right) W^{(j)^{\dagger}}\right) U_{2}^{\dagger}\right.\right. \\
& =2 \cdot U_{2} U_{1}\left(\sum_{j} p(j)|j\rangle\left\langle\left. j\right|^{J} \otimes \rho_{j}^{C} \otimes \mid 0\right\rangle\left\langle\left. 0\right|^{E} \otimes \xi_{a: n}^{D}\right) U_{1}^{\dagger} U_{2}^{\dagger}\right. \\
& =2 \cdot U^{J C} \otimes|0\rangle\left\langle\left. 0\right|^{E} \otimes \xi_{a: n}^{D}\right) U^{\dagger},
\end{aligned}
$$

where the inequality follows from Corollary 2.13, Eq. (2.20). 
Moreover, by the construction in Lemma 2.12 and Corollary 2.13 , for each $j$, the operator $\Pi^{(j)}$ is the projection operator onto the support of $W^{(j)}\left(\rho_{j}^{C} \otimes|0\rangle\left\langle\left. 0\right|^{E} \otimes \xi_{a: n}^{D}\right) W^{(j)^{\dagger}}\right.$. Hence, we have

$$
\begin{aligned}
\operatorname{Tr}\left[\widetilde{\Pi} U\left(\rho^{J C} \otimes|0\rangle\left\langle\left. 0\right|^{E} \otimes \xi_{a: n}^{D}\right) U^{\dagger}\right]\right. & =\operatorname{Tr}\left[\Pi^{\prime} U_{1}\left(\rho^{J C} \otimes|0\rangle\left\langle\left. 0\right|^{E} \otimes \xi_{a: n}^{D}\right) U_{1}^{\dagger}\right]\right. \\
& =\sum_{j} p(j) \operatorname{Tr}\left[\Pi^{(j)} W^{(j)}\left(\rho_{j}^{C} \otimes|0\rangle\left\langle\left. 0\right|^{E} \otimes \xi_{a: n}^{D}\right) W^{(j)^{\dagger}}\right]\right. \\
& =1 .
\end{aligned}
$$

This completes the proof.

Remark: In the above corollary, there is an implicit assumption that the eigenvalues of $\rho_{j}^{C}$ are rational. However, we can assume without loss of generality that this is always the case since the set of rational numbers is dense and the error due to this assumption can be made arbitrarily close to zero.

\section{Main result}

In this section, we prove our main result.

Theorem 3.1. Let $|\psi\rangle^{R A B C}$ be a pure quantum state shared between Referee $(R)$, Alice $(A C)$ and Bob $(B)$. For every $\epsilon_{1}, \epsilon_{2} \in(0,1)$ satisfying $\epsilon_{1}+9 \epsilon_{2} \leq 1$, there exists an entanglement-assisted one-way protocol operated by Alice and Bob which starts in the state $|\psi\rangle^{R A B C}$, and outputs a state $\phi^{R A B C} \in \mathrm{B}^{\epsilon_{1}+9 \epsilon_{2}}\left(\psi^{R A B C}\right)$ where registers $A, B C$ and $R$ are held by Alice, Bob and Referee, respectively. The communication cost of this protocol is upper bounded by

$$
\frac{1}{2} \inf _{\psi^{\prime} \in \mathrm{B}^{\epsilon_{1}}\left(\psi^{R B C}\right)} \inf _{\sigma \in \mathrm{ME}_{R-B-C}^{\epsilon_{2}^{4} / 4, \psi^{\prime}}}\left[\mathrm{D}_{\max }\left(\psi^{\prime R B C} \| \sigma^{R B C}\right)-\mathrm{D}_{\mathrm{H}}^{\epsilon_{2}^{2}}\left(\psi^{\prime B C} \| \sigma^{B C}\right)\right]+\log \frac{1}{\epsilon_{2}^{2}}+1 .
$$

Proof: Fix $\psi^{\prime R B C} \in \mathrm{B}^{\epsilon_{1}}\left(\psi^{R B C}\right)$ and $\sigma^{R B C} \in \mathrm{ME}_{R-B-C}^{\epsilon_{2}^{4} / 4, \psi^{\prime}}$. As explained in Section 2.2, there exists a decomposition of register $B$ as $\mathcal{H}^{B}=\bigoplus_{j} \mathcal{H}^{B_{j}^{R}} \otimes \mathcal{H}^{B_{j}^{C}}$ such that

$$
\psi^{\prime R B}=\sigma^{R B}=\bigoplus_{j} p(j){\psi^{\prime}}_{j}^{R B_{j}^{R}} \otimes{\psi^{\prime}}_{j}^{B_{j}^{C}}
$$

and

$$
\sigma^{R B C}=\bigoplus_{j} p(j) \sigma_{j}^{R B_{j}^{R}} \otimes \sigma_{j}^{B_{j}^{C} C}
$$

where $\sigma_{j}^{R B_{j}^{R}}={\psi^{\prime}}_{j}^{R B_{j}^{R}}, \sigma_{j}^{B_{j}^{C} C} \in \mathrm{B}^{\epsilon_{2}^{4} / 4}\left(\operatorname{Tr}_{B_{j}^{R}}\left(\left(\Pi_{j} \otimes \mathbb{1}\right) \psi^{\prime B C}\left(\Pi_{j} \otimes \mathbb{1}\right)\right)\right)$ and $\Pi_{j}$ is the projection operator over the $j$-th subspace of register $B$. If $\sigma^{R B C}=\psi^{\prime R B} \otimes \psi^{\prime C}$, Alice and Bob can redistribute $\psi^{R A B C}$ with error $9 \epsilon_{2}>0$ and communication cost bounded by Eq. (3.1) using the AJW protocol as explained in Theorem [2.11. However, in general, $\sigma^{R B C}$ is not necessarily a product state. In that case, our broad strategy is to let Alice and Bob transform $\psi^{\prime R B C}$ through a local unitary procedure which maps $\sigma^{R B C}$ to a product state. Then, they can use the AJW protocol to redistribute this new state. Before achieving this, Alice and Bob will perform some pre-processing on their shared state, as follows. 
i. Viewing $\sigma^{R B C}$ as a classical-quantum state: Let $B^{R}$ and $B^{C}$ be two quantum registers with $\left|B^{R}\right|=\max _{j}\left|B_{j}^{R}\right|$ and $\left|B^{C}\right|=\max _{j}\left|B_{j}^{C}\right|$. As a consequence of Eq. (3.3), there exists an isometry $U_{\mathrm{i}}: \mathcal{H}^{B} \rightarrow \mathcal{H}^{B^{R} J B^{C}}$ which takes $\sigma^{R B C}$ to the state $\sigma_{1}^{R B^{R} J B^{C} C}$ defined as

$$
\sigma_{1}^{R B^{R} J B^{C} C} \quad:=\sum_{j} p(j) \sigma_{j}^{R B^{R}} \otimes|j\rangle\left\langle\left. j\right|^{J} \otimes \sigma_{j}^{B^{C} C} .\right.
$$

Let $\left|\psi^{\prime}\right\rangle^{R A B C}$ be a purification of $\psi^{R B C}$ satisfying $\mathrm{P}\left(|\psi\rangle^{R A B C},\left|\psi^{\prime}\right\rangle^{R A B C}\right) \leq \epsilon_{1}$, as guaranteed by the Uhlmann theorem. Define

$$
\left|\psi_{1}\right\rangle^{R A B^{R} J B^{C} C}:=U_{\mathrm{i}}\left|\psi^{\prime}\right\rangle^{R A B C}=\sum_{j, j^{\prime}}|j\rangle\left\langle\left. j^{\prime}\right|^{J} \otimes \psi_{j, j^{\prime}}^{R A B^{R} B^{C} C} .\right.
$$

ii. Transferring $B^{C}$ from Bob to Alice without communication: Recall that register $J$ is classical in $\psi_{1}^{R B^{R} J B^{C}}$ and conditioned on $J, R B^{R}$ and $B^{C}$ are decoupled, that is

$$
\psi_{1}^{R B^{R} J B^{C}}=\sigma_{1}^{R B^{R} J B^{C}}=\sum_{j} p(j) \sigma_{j}^{R B^{R}} \otimes|j\rangle\left\langle\left. j\right|^{J} \otimes \sigma_{j}^{B^{C}} .\right.
$$

This implies that $\mathrm{I}\left(R B^{R}: B^{C} \mid J\right)_{\psi_{1}}=0$. So, Alice and Bob can use the folklore protocol for redistributing quantum Markov states explained in Fig. 2 and transfer $B^{C}$ to Alice, as follows:

Since Alice holds registers $A C$, she can prepare the following purification of $\psi_{1}^{R B^{R} J B^{C}}$ :

$$
\left|\psi_{1}^{\prime}\right\rangle^{R B^{R} J J^{\prime} B^{C} G H}=\sum_{j} \sqrt{p(j)}\left|\sigma_{j}\right\rangle^{R B^{R} G} \otimes|j, j\rangle^{J J^{\prime}} \otimes\left|\sigma_{j}\right\rangle^{B^{C} H}
$$

where registers $J^{\prime} G H$ are held by Alice. Let $\delta_{1} \in(0,1), n_{1}:=\left|B^{C} H\right|^{2 / \delta_{1}^{2}}$, and $D_{1}, D_{1}^{\prime}$ be registers with $\left|D_{1}\right|=\left|D_{1}^{\prime}\right|=n_{1}$. Conditioned on register $J$, Alice and Bob use the embezzling state $|\xi\rangle^{D_{1} D_{1}^{\prime}}$ (as defined in Eq. (2.10) ) and the inverse of the van Dam-Hayden protocol [27] to embezzle out $\left|\sigma_{j}\right\rangle^{B^{C} H}$ in superposition and obtain a state $\widetilde{\psi}_{1}$ such that

$$
\mathrm{P}\left(\widetilde{\psi}_{1}^{R B^{R} G J J^{\prime} D_{1} D_{1}^{\prime}}, \sum_{j} \sqrt{p(j)}\left|\sigma_{j}\right\rangle^{R B^{R} G} \otimes|j, j\rangle^{J J^{\prime}} \otimes|\xi\rangle^{D_{1} D_{1}^{\prime}}\right) \leq \delta_{1} .
$$

Finally, conditioned on register $J$, Alice locally generates $\left|\sigma_{j}\right\rangle^{B^{C} H}$ in superposition with registers $B^{C} H$ on her side, and applies an Uhlmann unitary operator to her registers in order to prepare the purification $\left|\psi_{1}\right\rangle^{R A B^{R} J B^{C} C}$. Let $U_{\mathrm{ii}, A}$ and $U_{\mathrm{ii}, B}$ denote the overall unitary operators applied by Alice and Bob, respectively, in this step. After applying $U_{\mathrm{ii}, A}$ and $U_{\mathrm{ii}, B}$, the global state is $\left|\psi_{2}\right\rangle$ satisfying

$$
\mathrm{P}\left(\psi_{2}^{R A B^{R} J B^{C} C D_{1} D_{1}^{\prime}},\left|\psi_{1}\right\rangle\left\langle\left.\psi_{1}\right|^{R A B^{R} J B^{C} C} \otimes \mid \xi\right\rangle\left\langle\left.\xi\right|^{D_{1} D_{1}^{\prime}}\right) \leq \delta_{1},\right.
$$

where registers $A B^{C} C$ are with Alice, registers $B^{R} J$ are with Bob and register $R$ is with Referee. Thus, the problem reduces to the case where the global state is $\left|\psi_{1}\right\rangle$ and the register $B^{C}$ belongs to Alice, up to a purified distance $\delta_{1}$. We will now assume that this is indeed the case and later, we will account for the inaccuracy introduced by this assumption in the error analysis of our protocol, using the data processing inequality. 
Suppose the global state is $\left|\psi_{1}\right\rangle^{R A B^{R} J B^{C} C}$ such that registers $A B^{C} C, B^{R} J$ and $R$ are held by Alice, Bob and Referee, respectively, and Alice wants to send $B^{C} C$ to Bob. To achieve this, we introduce a two-step unitary procedure which decouples registers $R B^{R} J$ and $B^{C} C$ in $\sigma_{1}^{R B^{R} J B^{C} C}$ while keeping the state of registers $R B^{R} J$ unchanged. This operation transforms $\sigma_{1}$ to a product state and allows us to use the AJW protocol as a subroutine to achieve the redistribution.

To decouple $R B^{R} J$ from $B^{C} C$ in $\sigma_{1}$, we use embezzlement and the unitary operator, given by Corollary 2.14. This unitary operator acts on registers $J B^{C} C$ and is read-only on register $J$. However, since register $J$ is not classical in $\psi_{1}^{R B^{R} J B^{C} C}$, it may disturb the marginal state $\psi_{1}^{R B^{R} J}$. This issue can be resolved by first coherently measuring register $J$ using an additional maximally entangled state. This operation transforms $\psi_{1}^{R B^{R} J B^{C} C}$ to a classical-quantum state, classical in register $J$, while keeps $\sigma_{1}^{R B^{R} J B^{C} C}$ intact. The following two steps contain the detailed construction of these two unitary procedures.

1. Coherent measurement of register $J$ : Let $F$ be a register with $|F|=|J|$, and $\{|f\rangle\}_{f=0}^{|F|-1}$ be a basis for $\mathcal{H}^{F}$. For $a, b \in\{0, \ldots,|F|-1\}$, let $P_{a, b} \in \mathrm{U}\left(\mathcal{H}^{F}\right)$ be the Heisenberg-Weyl operator defined as $P_{a, b}:=\sum_{f} \exp \left(\frac{2 \pi i f b}{|F|}\right)|f+a\rangle\left\langle\left. f\right|^{F}\right.$. Let $U_{1} \in \mathrm{U}\left(\mathcal{H}^{J F}\right)$ be a unitary operator defined as $U_{1}:=\sum_{j}|j\rangle\left\langle\left. j\right|^{J} \otimes P_{j, 0}^{F}\right.$. Define

$$
\left|\kappa_{1}\right\rangle^{R A B^{R} J B^{C} C F F^{\prime}}:=U_{1}\left(\left|\psi_{1}\right\rangle^{R A B^{R} J B^{C} C} \otimes|\Psi\rangle^{F F^{\prime}}\right)
$$

and

$$
\tau_{1}^{R B^{R} J B^{C} C F}:=\quad U_{1}\left(\sigma_{1}^{R B^{R} J B^{C} C} \otimes \frac{\mathbb{1}^{F}}{|F|}\right) U_{1}^{\dagger}
$$

where $|\Psi\rangle^{F F^{\prime}}$ is the maximally entangled state over registers $F$ and $F^{\prime}$. Notice that the set of Heisenberg-Weyl operators is closed under multiplication (up to global phases) and each $P_{a, b}$ is traceless unless $a=b=0$. Therefore, the unitary operator $U_{1}$ acts trivially on $\sigma_{1}$ while it measures register $J$ in $\psi_{1}^{R B^{R} J B^{C} C}$ coherently. In particular,

$$
\tau_{1}^{R B^{R} J B^{C} C F}=\sigma_{1}^{R B^{R} J B^{C} C} \otimes \frac{\mathbb{1}^{F}}{|F|}
$$

and

$$
\kappa_{1}^{R B^{R} J B^{C} C}=\sum_{j}|j\rangle\left\langle\left. j\right|^{J} \otimes \psi_{j, j}^{R B^{R} B^{C} C}\right.
$$

2. Decoupling registers $B^{C} C$ from $R B^{R} J$ in $\tau_{1}$ : By Eqs. (3.4) and (3.6), register $J$ is classical in $\tau_{1}^{R B^{R} J B^{C} C}$ and conditioned on $J$, registers $R B^{R}$ are decoupled from $B^{C} C$. Hence, we can decouple registers $B^{C} C$ from registers $R B^{R} J$ in $\tau_{1}$ using embezzling states and applying the unitary operator given in Corollary 2.14 ,

For $\gamma_{2} \in(0,1)$ chosen as in Corollary 2.14, let $a_{2}=\left|B^{C} C\right| / \gamma_{2}, n_{2}=a_{2}^{1 / \delta_{2}^{2}}$, and $D_{2}, D_{2}^{\prime}$ and $E_{2}$ be quantum registers with $\left|D_{2}\right|=\left|D_{2}^{\prime}\right| \geq n_{2}$ and $\left|E_{2}\right|=a_{2}$. According to Corollary 2.14, there 
exist a unitary operator $U_{2} \in \mathrm{U}\left(\mathcal{H}^{J B^{C} C E_{2} D_{2}}\right)$, read-only on register $J$, and a projection operator $\widetilde{\Pi} \in \operatorname{Pos}\left(\mathcal{H}^{J B^{C} C E_{2} D_{2}}\right)$ such that

$$
\begin{aligned}
& \mathrm{D}_{\max }\left(U_{2}\left(\tau_{1}^{R B^{R} J B^{C} C} \otimes|0\rangle\left\langle\left. 0\right|^{E_{2}} \otimes \xi_{a_{2}: n_{2}}^{D_{2}}\right) U_{2}^{\dagger} \| \tau_{1}^{R B^{R} J} \otimes \nu_{2}^{B^{C} C E_{2}} \otimes \xi_{1: n_{2}}^{D_{2}}\right)\right. \leq \log \left(1+15 \delta_{2}^{2}\right), \\
& \widetilde{\Pi}\left(\tau_{1}^{B^{R} J} \otimes \nu_{2}^{B^{C} C E_{2}} \otimes \xi_{1: n_{2}}^{D_{2}}\right) \widetilde{\Pi} \preceq 2 \cdot U_{2}\left(\tau_{1}^{R B^{R} J B^{C} C} \otimes|0\rangle\left\langle\left. 0\right|^{E_{2}} \otimes \xi_{a_{2}: n_{2}}^{D_{2}}\right) U_{2}^{\dagger},\right.
\end{aligned}
$$

and

$$
\operatorname{Tr}\left[\widetilde{\Pi} U_{2}\left(\tau_{1}^{R B^{R} J B^{C} C} \otimes|0\rangle\left\langle\left. 0\right|^{E_{2}} \otimes \xi_{a_{2}: n_{2}}^{D_{2}}\right) U_{2}^{\dagger}\right]=1,\right.
$$

where $\nu_{2}^{B^{C} C E_{1}}=\frac{1}{a_{2}} \sum_{r=1}^{a_{2}}|r\rangle\left\langle\left. r\right|^{B^{C} C E_{2}}\right.$. Define

$$
\tau_{2}^{R B^{R} J B^{C} C E_{2} D_{2}} \quad:=\quad U_{2}\left(\tau_{1}^{R B^{R} J B^{C} C} \otimes|0\rangle\left\langle\left. 0\right|^{E_{2}} \otimes \xi_{a_{2}: n_{2}}^{D_{2}}\right) U_{2}^{\dagger},\right.
$$

and

$$
\left|\kappa_{2}\right\rangle^{R A B^{R} J B^{C} C E_{2} D_{2} D_{2}^{\prime} F F^{\prime}}:=U_{2}\left(\left|\kappa_{1}\right\rangle^{R A B^{R} J B^{C} C} \otimes|0\rangle^{E_{2}} \otimes\left|\xi_{a_{2}: n_{2}}\right\rangle^{D_{2} D_{2}^{\prime}}\right)
$$

Since $U_{2}$ is read-only on register $J$ and $J$ is classical in the state $\kappa_{1}^{R B^{R} J B^{C} C}$, the unitary operator $U_{2}$ keeps $\kappa_{1}^{R B^{R} J}$ intact. So, we have

$$
\kappa_{2}^{R B^{R} J}=\kappa_{1}^{R B^{R} J}=\psi_{1}^{R B^{R} J} .
$$

Moreover, by Eq. (3.8), $\tau_{2}$ is close to a product state in max-relative entropy and therefore, we can claim the following statement, proved towards the end.

Claim 3.2. For the state $\kappa_{2}$ defined above, we have

$$
\mathrm{D}_{\max }\left(\kappa_{2}^{R B^{R} J B^{C} C E_{2} D_{2} F} \| \kappa_{2}^{R B^{R} J} \otimes \nu_{2}^{B^{C} C E_{2}} \otimes \xi_{1: n_{2}}^{D_{2}} \otimes \frac{\mathbb{1}^{F}}{|F|}\right) \leq \mathrm{D}_{\max }\left(\psi^{\prime R B C} \| \sigma^{R B C}\right)+5 \delta_{2}
$$

and

$$
\mathrm{D}_{\mathrm{H}}^{\epsilon_{2}^{2}}\left(\kappa_{2}^{B^{R} J B^{C} C E_{2} D_{2} F} \| \kappa_{2}^{B^{R} J} \otimes \nu_{2}^{B^{C} C E_{2}} \otimes \xi_{1: n_{2}}^{D_{2}} \otimes \frac{\mathbb{1}^{F}}{|F|}\right) \geq \mathrm{D}_{\mathrm{H}}^{\epsilon_{2}^{4} / 4}\left(\psi^{\prime B C} \| \sigma^{B C}\right)-1 .
$$

Using the claim, we proceed as follows. Let

$$
\beta:=\mathrm{D}_{\max }\left(\psi^{R B C} \| \sigma^{R B C}\right)+5 \delta_{2},
$$

and $S$ and $T$ be quantum registers such that $|S|=|T|=\left|B^{C} C E_{2} D_{2}\right|$. Let $|\eta\rangle^{S T}$ be a purification of $\nu_{2}^{B^{C} C E_{2}} \otimes \xi_{1: n_{2}}^{D_{2}} \otimes \frac{\mathbb{1}^{F}}{|F|}$ such that $\eta^{T}=\nu_{2}^{B^{C} C E_{2}} \otimes \xi_{1: n_{2}}^{D_{2}} \otimes \frac{\mathbb{1}^{F}}{|F|}$.

To redistribute registers $B^{C} C$ in the state $\psi_{1}$ with the desired cost, Claim 3.2 suggests that it would be sufficient for parties to transform their joint state $\psi_{1}$ to $\kappa_{2}$ through the unitary operators $U_{2} U_{1}$, then use the AJW protocol to redistribute registers $B^{C} C E_{2} D_{2} F$, and finally, transform back $\kappa_{2}$ to 
the state $\psi_{1}$ by applying $U_{1}^{-1} U_{2}^{-1}$. However, in order to apply $U_{2} U_{1}$, one needs to have access to all the registers $J B^{C} C$, but initially registers $B^{C} C$ are with Alice and register $J$ is with Bob. This problem can be resolved using the Uhlmann theorem. Recall that $\kappa_{2}^{R B^{R} J}=\psi_{1}^{R B^{R} J}$ as mentioned in Eq. (3.11). Therefore, there exists an Uhlmann isometry $V: \mathcal{H}^{A B^{C} C} \rightarrow \mathcal{H}^{A C B^{C} E_{2} D_{2} D_{2}^{\prime} F F^{\prime}}$ such that

$$
V\left|\psi_{1}\right\rangle^{R A B^{R} J B^{C} C}=\left|\kappa_{2}\right\rangle^{R A B^{R} J B^{C} C E_{2} D_{2} D_{2}^{\prime} F F^{\prime}}
$$

Notice that $V$ only acts on registers $A B^{C} C$ which are initially with Alice and so Alice can apply the isometry $V$ locally to transform $\psi_{1}$ to $\kappa_{2}$.

Now, we have all the ingredients for the protocol and we proceed to construct the protocol as follows:

The protocol. In order to redistribute $|\psi\rangle^{R A B C}$, Alice and Bob run the following protocol.

1. Initially, Alice and Bob start in the state $|\psi\rangle^{R A B C}$ and pre-share quantum states $\left|\xi^{\prime}\right\rangle^{X^{\prime} X}$, $\left|\xi_{a_{2}: n_{2}}\right\rangle^{D_{2}^{\prime} D_{2}}$ and $m:=\left\lceil\frac{2^{\beta}}{\epsilon_{2}^{2}}\right]$ copies of the state $|\eta\rangle^{S T}$. Hence, the initial joint quantum state of Referee, Alice and Bob is

$$
|\psi\rangle^{R A B C} \otimes|\xi\rangle^{D_{1}^{\prime} D_{1}} \otimes\left|\xi_{a_{2}: n_{2}}\right\rangle^{D_{2}^{\prime} D_{2}} \bigotimes_{i=1}^{m}|\eta\rangle^{S_{i} T_{i}}
$$

such that register $R$ is held by Referee, registers $\left(A C S_{1} \ldots S_{m} D_{1}^{\prime} D_{2}^{\prime}\right)$ are held by Alice and registers $\left(B T_{1} \ldots T_{m} D_{1} D_{2}\right)$ are held by Bob.

2. Bob prepares ancilla qubits $|0\rangle^{E_{1}}$ and applies the isometry $U_{\mathrm{ii}, B} U_{\mathrm{i}}$ on his registers, and Alice applies the isometry $V U_{\mathrm{ii}, A}$ on her registers. Hence, their joint state transforms into a quantum state, say $\omega$, which has purified distance at most $\delta_{1}$ from $\left|\kappa_{2}\right\rangle^{R A B^{R} J B^{C} C E_{2} D_{2} D_{2}^{\prime} F F^{\prime}}$ such that registers $\left(A B^{C} C D_{1}^{\prime} E_{2} D_{2} D_{2}^{\prime} F F^{\prime}\right)$ are with Alice, registers $\left(B^{R} J B^{C} E_{1} D_{1}\right)$ are with Bob and register $(R)$ is with Referee.

3. Running the protocol given by Theorem 2.11, parties redistribute their registers assuming their joint state is $\left|\kappa_{2}\right\rangle^{R A B^{R} J B^{C} C E_{2} D_{2} D_{2}^{\prime} F F^{\prime}}$ and using the shared entangled state $\bigotimes_{i=1}^{m}|\eta\rangle^{S_{i} T_{i}}$, and end up in the state $\hat{\omega}^{R A B^{R} J B^{C} C E_{2} D_{2} D_{2}^{\prime} F F^{\prime}}$ such that register $(R)$ is held with Referee, $\left(A D_{2}^{\prime} F^{\prime}\right)$ are held with Alice and $\left(B^{R} J B^{C} C E_{2} D_{2} F\right)$ are held with Bob.

4. Bob applies the operator $\left(U_{2} U_{1} U_{\mathrm{i}}\right)^{-1}$ on registers $B^{R} J B^{C} C E_{2} D_{2} F$.

5. The final state is obtained in registers $R A B C$.

According to Theorem 2.11, the communication cost of this protocol is

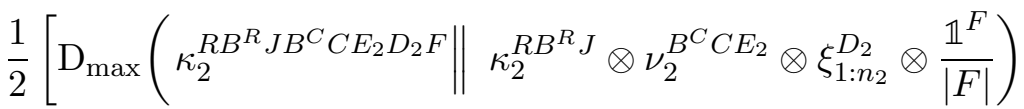

$$
\begin{aligned}
& \left.-\mathrm{D}_{\mathrm{H}}^{\epsilon_{2}^{2}}\left(\kappa_{2}^{B^{R} J B^{C} C E_{2} D_{2} F} \| \kappa_{2}^{B^{R} J} \otimes \nu_{2}^{B^{C} C E_{2}} \otimes \xi_{1: n_{2}}^{D_{2}} \otimes \frac{\mathbb{1}^{F}}{|F|}\right)\right]+\log \frac{1}{\epsilon_{2}^{2}}
\end{aligned}
$$


which is at most

$$
\frac{1}{2}\left[\mathrm{D}_{\max }\left(\psi^{\prime R B C} \| \sigma^{R B C}\right)-\mathrm{D}_{\mathrm{H}}^{\epsilon_{2}^{4} / 4}\left(\psi^{\prime B C} \| \sigma^{B C}\right)\right]+2.5 \delta_{2}+\log \frac{1}{\epsilon_{2}^{2}}+1,
$$

by Claim 3.2 ,

Correctness of the protocol. Let $\phi$ be the final joint state of parties in the above protocol. We have

$$
\begin{aligned}
& \mathrm{P}\left(\phi^{R A B C}, \psi^{R A B C}\right) \\
& \leq \mathrm{P}\left(\phi^{R A B C}, \psi^{\prime R A B C}\right)+\mathrm{P}\left(\psi^{\prime R A B C}, \psi^{R A B C}\right) \\
& \leq \mathrm{P}\left(\phi^{R A B C E_{2} D_{2} D_{2}^{\prime} F F^{\prime}}, \psi^{\prime R A B C} \otimes|0\rangle\left\langle\left. 0\right|^{E_{2}} \otimes \xi_{a_{2}: n_{2}}^{D_{2} D_{2}^{\prime}} \otimes \mid \Psi\right\rangle\left\langle\left.\Psi\right|^{F F^{\prime}}\right)+\epsilon_{1}\right. \\
& \leq \mathrm{P}\left(\hat{\omega}^{R A B^{R} J B^{C} C E_{2} D_{2} D_{2}^{\prime} F F^{\prime}}, \kappa_{2}^{R A B^{R} J B^{C} C E_{2} D_{2} D_{2}^{\prime} F F^{\prime}}\right)+\epsilon_{1} \\
& \leq \mathrm{P}\left(\hat{\omega}^{R A B^{R} J B^{C} C E_{2} D_{2} D_{2}^{\prime} F F^{\prime}}, \omega^{R A B^{R} J B^{C} C E_{2} D_{2} D_{2}^{\prime} F F^{\prime}}\right) \\
& \quad+\mathrm{P}\left(\omega^{R A B^{R} J B^{C} C E_{2} D_{2} D_{2}^{\prime} F F^{\prime}}, \kappa_{2}^{R A B^{R} J B^{C} C E_{2} D_{2} D_{2}^{\prime} F F^{\prime}}\right)+\epsilon_{1} \\
& \leq \quad \epsilon_{1}+9 \epsilon_{2}+\delta_{1} .
\end{aligned}
$$

where the second and third inequalities follow from monotonicity of purified distance under quantum operations and the last inequality holds since $\hat{\omega} \in \mathrm{B}^{9 \epsilon_{2}}(\omega)$ by Theorem 2.11, and $\omega \in \mathrm{B}^{\delta_{1}}\left(\kappa_{2}\right)$.

By construction of embezzling states, we can choose $\delta_{1}$ and $\delta_{2}$ arbitrarily small by allowing arbitrary large shared entanglement between Alice and Bob. Hence, the statement of the theorem follows.

It only remains to prove Claim 3.2 .

Proof of Claim [3.2; Consider the states and operators defined in the proof of Theorem 3.1, Since register $J$ is classical in both $\kappa_{1}^{R B^{R} J B^{C} C}$ and $\tau_{1}^{R B^{R} J B^{C} C}$ and $U_{2}$ is read-only on $J$, we have that $\kappa_{2}^{R B^{R} J}=\tau_{2}^{R B^{R} J}=\tau_{1}^{R B^{R} J}$, and $\kappa_{2}^{J B^{C} C E_{2} D_{2}} \in \mathrm{B}^{\epsilon_{2}^{4} / 4}\left(\tau_{2}^{J B^{C} C E_{2} D_{2}}\right)$. Therefore, we get

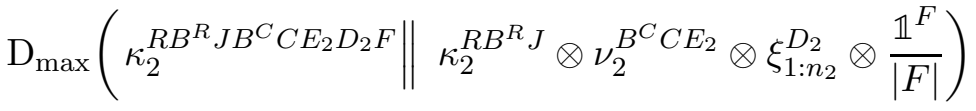

$$
\begin{aligned}
& \leq \mathrm{D}_{\max }\left(\kappa_{2}^{R B^{R} J B^{C} C E_{2} D_{2} F} \| \tau_{2}^{R B^{R} J B^{C} C E_{2} D_{2}} \otimes \frac{\mathbb{1}^{F}}{|F|}\right) \\
& +\mathrm{D}_{\max }\left(\tau_{2}^{R B^{R} J B^{C} C E_{2} D_{2}} \| \tau_{2}^{R B^{R} J} \otimes \nu_{2}^{B^{C} C E_{2}} \otimes \xi_{1: n_{2}}^{D_{2}}\right) \\
& \leq \mathrm{D}_{\max }\left(\psi^{\prime R B C} \| \sigma^{R B C}\right)+\log \left(1+15 \delta_{2}^{2}\right) \text {, }
\end{aligned}
$$

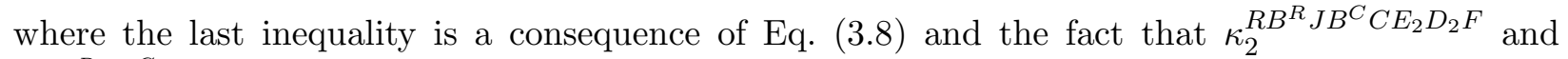
$\tau_{2}^{R B^{R} J B^{C} C E_{2} D_{2} F}$ are unitary transformations of $\psi^{\prime R B C}$ and $\sigma^{R B C}$, respectively. The above equation implies Eq. (3.12) since $\log _{2}\left(1+15 x^{2}\right) \leq 5 x$ for all $x \geq 0$. 
In the rest of the proof, we show that

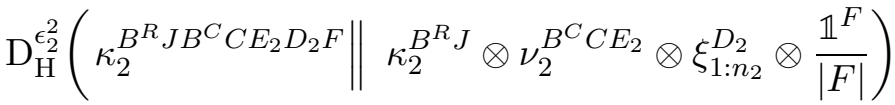

$$
\begin{aligned}
& \geq \quad \mathrm{D}_{\mathrm{H}}^{\epsilon_{2}^{4} / 4}\left(\kappa_{2}^{B^{R} J B^{C} C E_{2} D_{2} F} \| \tau_{2}^{B^{R} J B^{C} C E_{2} D_{2}} \otimes \frac{\mathbb{1}^{F}}{|F|}\right)-1 .
\end{aligned}
$$

Then, Eq. (3.13) follows since $\kappa_{2}^{R B^{R} J B^{C} C E_{2} D_{2} F}$ and $\tau_{2}^{R B^{R} J B^{C} C E_{2} D_{2} F}$ are unitary transformations of $\psi^{\prime R B C}$ and $\sigma^{R B C}$, respectively. Let $\lambda:=\mathrm{D}_{\mathrm{H}}^{\epsilon_{2}^{4} / 4}\left(\kappa_{2}^{B^{R} J B^{C} C E_{2} D_{2} F} \| \tau_{2}^{B^{R} J B^{C} C E_{2} D_{2} F}\right)$, and $\Pi^{\prime}$ be the POVM operator achieving $\lambda$, i.e.,

$$
\operatorname{Tr}\left[\Pi^{\prime} \kappa_{2}^{\left.B^{R} J B^{C} C E_{2} D_{2} F\right]} \geq 1-\frac{\epsilon_{2}^{4}}{4}\right.
$$

and

$$
\operatorname{Tr}\left[\Pi^{\prime}\left(\tau_{2}^{B^{R} J B^{C} C E_{2} D_{2}} \otimes \frac{\mathbb{1}^{F}}{|F|}\right)\right]=2^{-\lambda} .
$$

Recall that $\kappa_{2}^{B^{R} J}=\tau_{2}^{B^{R} J}=\tau_{1}^{B^{R} J}$. So, Eq. (3.9) implies that

$$
\widetilde{\Pi}\left(\kappa_{2}^{B^{R} J} \otimes \nu_{2}^{B^{C} C E_{2}} \otimes \xi_{1: n_{2}}^{D_{2}}\right) \widetilde{\Pi} \preceq 2 \cdot \tau_{2}^{B^{R} J B^{C} C E_{2} D_{2}} .
$$

Since $\sigma^{R B C} \in \mathrm{ME}_{R-B-C}^{\epsilon, \psi^{\prime}}$, the state $\kappa_{2}^{J B^{C} C E_{2} D_{2}}$ is $\left(\epsilon_{2}^{4} / 4\right)$-close to $\tau_{2}^{J B^{C} C E_{2} D_{2}}$ in purified distance. This implies that

$$
\operatorname{Tr}\left[\widetilde{\Pi} \kappa_{2}^{B^{R} J B^{C} C E_{2} D_{2} F}\right] \geq \operatorname{Tr}\left[\widetilde{\Pi} \tau_{2}^{B^{R} J B^{C} C E_{2} D_{2} F}\right]-\frac{\epsilon_{2}^{4}}{4}=1-\frac{\epsilon_{2}^{4}}{4}
$$

using Theorem 2.1, Theorem 2.3 and Eq. (3.10). So, the Gentle Measurement lemma, Lemma 2.2, implies that

$$
\left\|\frac{\widetilde{\Pi} \kappa_{2}^{B^{R} J B^{C} C E_{2} D_{2} F} \widetilde{\Pi}}{\operatorname{Tr}\left[\widetilde{\Pi} \kappa_{2}^{B^{R} J B^{C} C E_{2} D_{2} F}\right]}-\kappa_{2}^{B^{R} J B^{C} C E_{2} D_{2} F}\right\|_{1} \leq \epsilon_{2}^{2} .
$$

Define the POVM operator $\Pi:=\widetilde{\Pi} \Pi^{\prime} \widetilde{\Pi}$. By Eq. (3.2) and Eq. (3.19), we have

$$
\begin{aligned}
\operatorname{Tr}\left[\Pi \kappa_{2}^{\left.B^{R} J B^{C} C E_{2} D_{2} F\right]}\right. & =\operatorname{Tr}\left[\Pi^{\prime} \widetilde{\Pi} \kappa_{2}^{B^{R} J B^{C} C E_{2} D_{2} F} \widetilde{\Pi}\right] \\
& \geq\left(1-\frac{\epsilon_{2}^{4}}{4}\right)\left(\operatorname{Tr}\left[\Pi^{\prime} \kappa_{2}^{B^{R} J B^{C} C E_{2} D_{2} F}\right]-\frac{\epsilon_{2}^{2}}{2}\right) \quad \text { (By Theorem 2.1) } \\
& \geq 1-\epsilon_{2}^{2},
\end{aligned}
$$

and by Eq. (3.18), we get

$$
\begin{aligned}
\operatorname{Tr}\left[\Pi\left(\kappa_{2}^{B^{R} J} \otimes \nu_{2}^{B^{C} C E_{2}} \otimes \xi_{1: n_{2}}^{D_{2}} \otimes \frac{\mathbb{1}^{F}}{|F|}\right)\right] & \leq 2 \cdot \operatorname{Tr}\left[\Pi^{\prime}\left(\tau_{2}^{B^{R} J B^{C} C E_{2} D_{2}} \otimes \frac{\mathbb{1}^{F}}{|F|}\right)\right] \\
& =2^{-\lambda+1}
\end{aligned}
$$

which imply Eq. (3.15), as desired. 


\section{Asymptotic and i.i.d. analysis}

Suppose that the state $|\psi\rangle^{R^{n} A^{n} B^{n} C^{n}}=\left(|\psi\rangle^{R A B C}\right)^{\otimes n}$ is shared between Referee $\left(R^{n}\right)$, Alice $\left(A^{n} C^{n}\right)$ and $\operatorname{Bob}\left(B^{n}\right)$ where $R^{n}, A^{n}, B^{n}$ and $C^{n}$ denote $n$-fold tensor product of registers $R, A, B$ and $C$, respectively. Let $\epsilon:=\epsilon_{1}=\epsilon_{2}^{4} / 4$. By Theorem 3.1 and choosing $\sigma^{R^{n} B^{n} C^{n}}=\psi^{\prime R^{n} B^{n}} \otimes \psi^{C^{n}}$, there exists

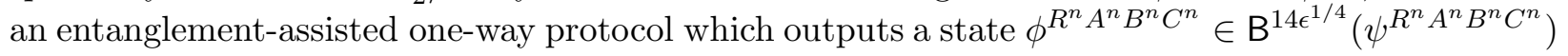
with communication cost $Q(n, \epsilon)$ at most

$$
\begin{aligned}
& Q(n, \epsilon) \\
& \leq \frac{1}{2} \inf _{\psi^{\prime} \in \mathrm{B}^{\epsilon}\left(\psi^{R^{n} B^{n} C^{n}}\right)}\left[\mathrm{D}_{\max }\left(\psi^{\prime R^{n} B^{n} C^{n}} \| \psi^{\prime R^{n} B^{n}} \otimes \psi^{C^{n}}\right)-\mathrm{D}_{\mathrm{H}}^{\epsilon}\left(\psi^{\prime B^{n} C^{n}} \| \psi^{\prime B^{n}} \otimes \psi^{C^{n}}\right)\right]+\log \frac{1}{2 \sqrt{\epsilon}} \\
& \leq \frac{1}{2} \inf _{\psi^{\prime} \in \mathrm{B}^{\epsilon}\left(\psi^{\left.R^{n} B^{n} C^{n}\right)}\right.}\left[\mathrm{D}_{\max }\left(\psi^{\prime R^{n} B^{n}} R^{n} B^{n} C^{n} \| \psi^{n} B^{R^{n} B^{n}} \otimes \psi^{C^{n}}\right)-\mathrm{D}_{\mathrm{H}}^{\epsilon}\left(\psi^{\prime B^{n} C^{n}} \| \psi^{B^{n}} \otimes \psi^{C^{n}}\right)\right]+\log \frac{1}{2 \sqrt{\epsilon}} \\
& \leq \frac{1}{2} \inf _{\substack{\psi^{\prime} \in \mathrm{B}^{\epsilon}\left(\psi^{R^{n}} B^{n} C^{n}\right) \\
\psi^{\prime R^{n} B^{n}}=\psi^{R^{n} B^{n}}}}\left[\mathrm{D}_{\max }\left(\psi^{R^{n} B^{n} C^{n}} \| \psi^{R^{n} B^{n}} \otimes \psi^{C^{n}}\right)-\mathrm{D}_{\mathrm{H}}^{2 \epsilon}\left(\psi^{B^{n} C^{n}} \| \psi^{B^{n}} \otimes \psi^{C^{n}}\right)\right]+\log \frac{1}{2 \sqrt{\epsilon}} \\
& \leq \frac{1}{2}\left[\mathrm{D}_{\max }^{\epsilon / 3}\left(\psi^{R^{n} B^{n} C^{n}} \| \psi^{R^{n} B^{n}} \otimes \psi^{C^{n}}\right)-\mathrm{D}_{\mathrm{H}}^{2 \epsilon}\left(\psi^{B^{n} C^{n}} \| \psi^{B^{n}} \otimes \psi^{C^{n}}\right)\right]+\log \frac{1}{2 \sqrt{\epsilon}}+\log \frac{72+\epsilon^{2}}{\epsilon^{2}},
\end{aligned}
$$

where the first inequality follows from Eq.(3.1), the third inequality follows from the definition of Hypothesis testing entropy, and the last inequality follows from Theorem 2.7 for the choice of $\epsilon, \delta \leftarrow \epsilon / 3, \rho^{A B} \leftarrow \psi^{R^{n} B^{n} C^{n}}, \rho^{A} \leftarrow \psi^{R^{n} B^{n}}$ and $\sigma^{B} \leftarrow \psi^{C^{n}}$. Therefore, using Theorem 2.6, the asymptotic communication rate of redistributing $n$ copies of a pure state $|\psi\rangle^{R A B C}$ is

$$
\lim _{n \rightarrow \infty} \frac{1}{n} Q(n, \epsilon) \leq \frac{1}{2} \mathrm{I}(R: C \mid B)_{\psi} .
$$

\section{Conclusion and outlook}

In this article, we revisited the task of one-shot quantum state redistribution, and introduced a new protocol achieving this task with communication cost

$$
\frac{1}{2} \min _{\psi^{\prime} \in \mathrm{B}^{\epsilon}\left(\psi^{R B C}\right)} \min _{\sigma^{R B C} \in \mathrm{ME}_{R-B-C}^{\epsilon, \psi^{\prime}}}\left[\mathrm{D}_{\max }\left(\psi^{R B C} \| \sigma^{R B C}\right)-\mathrm{D}_{\mathrm{H}}^{\epsilon}\left(\psi^{\prime B C} \| \sigma^{B C}\right)\right]+O\left(\log \frac{1}{\epsilon}\right),
$$

with error parameter $\epsilon$. This is the first result connecting the communication cost of state redistribution with Markov chains and it provides an operational interpretation for a one-shot representation of quantum conditional mutual information as explained in Sec 1. In the special case where $\psi^{R B C}$ is a quantum Markov chain, our protocol leads to near-zero communication which was not known for the previous protocols. Moreover, the communication cost of our protocol is lower than all previously known one-shot protocols and we show that it achieves the optimal cost of $\mathrm{I}(R: C \mid B)$ in the asymptotic i.i.d. setting. Our protocol also achieves the near-optimal result of Ref. [4] in the case when $\psi^{R B C}$ is classical.

A question of interest is whether the communication cost of our one-shot protocol can be bounded with $\mathrm{I}(R: C \mid B)$. In the quantum communication complexity setting, such a bound would imply 
the possibility of compressing the communication of bounded-round quantum protocols to their information content which would lead to a direct-sum theorem for bounded-round quantum communication complexity [25].

Another question that we have not addressed in this article is whether our bound is near-optimal. There are several known lower bounds in the literature for the communication cost of entanglementassisted quantum state redistribution, such as in Ref. [9] and Ref. [16]. However, it is not clear if our bound achieves any of them. Near-optimal bound for one-shot quantum state redistribution is a major open question.

\section{References}

[1] Anurag Anshu, Mario Berta, Rahul Jain, and Marco Tomamichel. Partially smoothed information measures. IEEE Transactions on Information Theory, 66(8):5022-5036, 2020.

[2] Anurag Anshu, Vamsi Krishna Devabathini, and Rahul Jain. Quantum communication using coherent rejection sampling. Physical Review Letters, 119:120506, September 2017.

[3] Anurag Anshu and Rahul Jain. Efficient methods for one-shot quantum communication. Technical Report arXiv:1809.07056 [quant-ph], arXiv.org, https://arxiv.org/abs/1809.07056, September 2018.

[4] Anurag Anshu, Rahul Jain, and Naqueeb A. Warsi. A unified approach to source and message compression. Technical Report arXiv:1707.03619 [quant-ph], arXiv.org, https://arxiv.org/pdf/1707.03619.pdf, July 2017.

[5] Anurag Anshu, Rahul Jain, and Naqueeb A. Warsi. A one-shot achievability result for quantum state redistribution. IEEE Transactions on Information Theory, 64(3):1425-1435, March 2018.

[6] Anurag Anshu, Rahul Jain, and Naqueeb A. Warsi. Building blocks for communication over noisy quantum networks. IEEE Transactions on Information Theory, 65(2):1287-1306, February 2019.

[7] Anurag Anshu, Rahul Jain, and Naqueeb Ahmad Warsi. A one-shot achievability result for quantum state redistribution. IEEE Transactions on Information Theory, 64(3):1425-1435, 2017.

[8] Koenraad M. R. Audenaert, Milan Mosonyi, and Frank Verstraete. Quantum state discrimination bounds for finite sample size. Journal of Mathematical Physics, 53(12):122205, 2012.

[9] Mario Berta, Mathias Christandl, and Dave Touchette. Smooth entropy bounds on one-shot quantum state redistribution. IEEE Transactions on Information Theory, 62(3):1425-1439, March 2016.

[10] Mario Berta, Kaushik P. Seshadreesan, and Mark M. Wilde. Rényi generalizations of the conditional quantum mutual information. Journal of Mathematical Physics, 56(2):022205, 2015.

[11] Matthias Christandl, Norbert Schuch, and Andreas Winter. Entanglement of the antisymmetric state. Communications in Mathematical Physics, 311(2):397-422, April 2012. 
[12] Nikola Ciganovic, Normand J. Beaudry, and Renato Renner. Smooth max-information as one-shot generalization for mutual information. IEEE Transactions on Information Theory, 60(3):1573-1581, 2014.

[13] Alexei Gilchrist, Nathan K. Langford, and Michael A. Nielsen. Distance measures to compare real and ideal quantum processes. Physical Review A, 71:062310, June 2005.

[14] Patrick Hayden, Richard Jozsa, Dénes Petz, and Andreas Winter. Structure of states which satisfy strong subadditivity of quantum entropy with equality. Communications in Mathematical Physics, 246(2):359-374, April 2004.

[15] Ben Ibinson, Noah Linden, and Andreas Winter. Robustness of quantum Markov chains. Communications in Mathematical Physics, 277(2):289-304, January 2008.

[16] Felix Leditzky, Mark M. Wilde, and Nilanjana Datta. Strong converse theorems using Rényi entropies. Journal of Mathematical Physics, 57(8):082202, 2016.

[17] Ke Li. Second-order asymptotics for quantum hypothesis testing. Annals of Statistics, 42(1):171-189, February 2014.

[18] Lorenzo Mascheroni. Adnotationes ad calculum integralem Euleri: in quibus nonnulla problemata. Galeatii, 1790.

[19] Tomohiro Ogawa and Hiroshi Nagaoka. Making good codes for classical-quantum channel coding via quantum hypothesis testing. IEEE Transactions on Information Theory, 53(6):2261$2266,2007$.

[20] Marco Tomamichel. A Framework for Non-Asymptotic Quantum Information Theory. PhD thesis, Eidgenössische Technische Hochschule (ETH), Zürich, 2012. Diss. Nr. 20213.

[21] Marco Tomamichel. Quantum Information Processing with Finite Resources: Mathematical Foundations, volume 5. Springer, 2015.

[22] Marco Tomamichel, Roger Colbeck, and Renato Renner. A fully quantum asymptotic equipartition property. IEEE Transactions on Information Theory, 55(12):5840-5847, 2009.

[23] Marco Tomamichel, Roger Colbeck, and Renato Renner. Duality between smooth min-and max-entropies. IEEE Transactions on Information Theory, 56(9):4674-4681, 2010.

[24] Marco Tomamichel and Masahito Hayashi. A hierarchy of information quantities for finite block length analysis of quantum tasks. IEEE Transactions on Information Theory, 59(11):7693$7710,2013$.

[25] Dave Touchette. Quantum information complexity. In Proceedings of the Forty-Seventh Annual ACM on Symposium on Theory of Computing, STOC '15, pages 317-326, New York, NY, USA, 2015. ACM.

[26] Armin Uhlmann. The "transition probability" in the state space of a *algebra. Reports on Mathematical Physics, 9(2):273 - 279, 1976.

[27] Wim van Dam and Patrick Hayden. Universal entanglement transformations without communication. Physical Review A, 67:060302, June 2003.

[28] John Watrous. The Theory of Quantum Information. Cambridge University Press, May 2018. 
[29] Andreas Winter. Coding theorem and strong converse for quantum channels. IEEE Transactions on Information Theory, 45(7):2481-2485, 1999. 